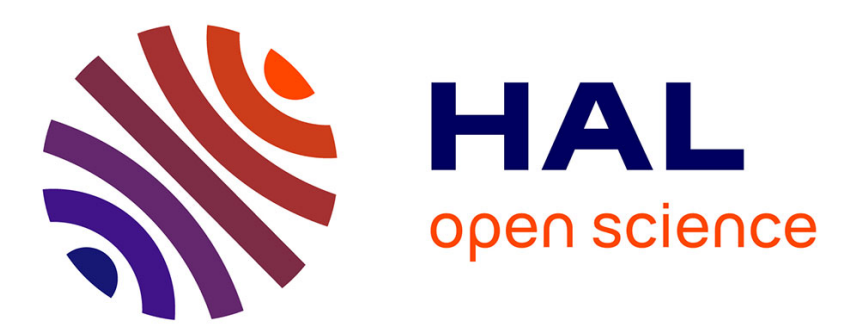

\title{
Syndromes de chevauchement asthme-BPCO (ACOS) : étude de la prévalence et des caractéristiques des ACOS parmi la cohorte de BPCO du CHU d'Amiens
}

\author{
Mélanie Drucbert
}

\section{- To cite this version:}

Mélanie Drucbert. Syndromes de chevauchement asthme-BPCO (ACOS) : étude de la prévalence et des caractéristiques des ACOS parmi la cohorte de BPCO du CHU d'Amiens. Pneumologie et système respiratoire. 2018. dumas-02131941

\section{HAL Id: dumas-02131941 https://dumas.ccsd.cnrs.fr/dumas-02131941}

Submitted on 16 May 2019

HAL is a multi-disciplinary open access archive for the deposit and dissemination of scientific research documents, whether they are published or not. The documents may come from teaching and research institutions in France or abroad, or from public or private research centers.
L'archive ouverte pluridisciplinaire HAL, est destinée au dépôt et à la diffusion de documents scientifiques de niveau recherche, publiés ou non, émanant des établissements d'enseignement et de recherche français ou étrangers, des laboratoires publics ou privés. 
UNIVERSITE DE PICARDIE JULES VERNE

FACULTE DE MEDECINE D’AMIENS

2018

\section{SYNDROMES DE CHEVAUCHEMENT ASTHME-BPCO (ACOS) : \\ ETUDE DE LA PREVALENCE ET DES CARACTERISTIQUES DES ACOS PARMI LA COHORTE DE BPCO DU CHU D'AMIENS}

THESE POUR LE DOCTORAT EN MEDECINE (DIPLOME D'ETAT)

SPECIALITE PNEUMOLOGIE

PRESENTEE ET SOUTENUE PUBLIQUEMENT

LE 10 OCTOBRE 2018

PAR

MELANIE DRUCBERT

Thèse $n^{\circ} 2018-150$

PRESIDENT DU JURY : $\quad$ Monsieur le Professeur Vincent JOUNIEAUX

JUGES : $\quad$ Monsieur le Professeur Michel ANDREJAK

Monsieur le Professeur Jean SCHMIDT

Madame le Docteur Brigitte GUBLER

DIRECTRICE : $\quad$ Madame le Professeur Claire ANDREJAK 
Remerciements

\section{A Monsieur le Professeur Vincent JOUNIEAUX}

Professeur des Universités-Praticien Hospitalier

(Pneumologie)

Chef du Service de Pneumologie, Pôle "Cœur - Thorax - Vaisseaux"

Chef du Service de Réanimation Respiratoire, Pôle « Anesthésie - Réanimations »

Vous me faites l'honneur de juger et présider cette thèse. Soyez assuré de ma plus profonde reconnaissance et de mon plus sincère respect.

Vous m'avez transmis vos connaissances et votre passion pour la pneumologie, de l'externat à l'internat.

Vous m'avez apporté écoute et soutien dans mes projets et je suis très touchée de la confiance que vous m'accordez.

Pour cela, je vous remercie et espère que ce travail sera à la hauteur de vos attentes. 


\section{A Monsieur le Professeur Michel ANDREJAK}

Professeur des Universités-Praticien Hospitalier consultant

(Pharmacologie fondamentale clinique)

Ancien Directeur du Centre Régional de Pharmacovigilance d'Amiens

Ancien Responsable du service de pharmacologie clinique

Pôle Biologie, Pharmacie et Santé des populations

Officier dans l'Ordre des Palmes Académiques

Vous me faites l'honneur de juger ce travail.

Veuillez accepter mes sincères remerciements et mon profond respect. 


\section{A Madame le Professeur Claire ANDREJAK \\ Professeur des Universités - Praticien Hospitalier \\ (Pneumologie)}

Tu me fais l'honneur d'avoir accepté la direction de ce travail.

Merci pour ton aide précieuse, tes conseils et ta disponibilité tout au long de ce travail. J'espère avoir répondu à tes attentes.

Je te remercie aussi pour tout ce que tu m'as transmis au cours de l'internat. Malgré la charge de tes activités, tu as toujours été présente et as su nous transmettre tes connaissances. 


\section{A Monsieur le Professeur Jean SCHMIDT \\ Professeur des Universités - Praticien Hospitalier \\ (Médecine interne)}

Vous me faites l'honneur de juger ce travail. Soyez assuré de ma profonde reconnaissance.

Je suis sortie de médecine interne enrichie de connaissances et d'un regard nouveau sur ma spécialité et la médecine.

Merci pour tout ce que vous m'avez transmis. 


\section{A Madame le Docteur Brigitte GUBLER}

Maître de Conférences-Praticien Hospitalier

(Immunologie)

Responsable du Centre d'Activité « Oncobiologie moléculaire »

Pôle «Biologie, pharmacie et santé des populations

Vous me faites l'honneur de juger ce travail et d'avoir accepté de participer à ce jury de thèse.

Veuillez accepter mes sincères remerciements et mon profond respect. 
Merci également :

A l'ensemble des médecins du service de Pneumologie d'Amiens : Claire, Eline, Damien, Géraldine, Pierre-Alexandre, Julien, Isabelle, Bénédicte, Mme Popin, Jean-Baptiste, Elodie... Merci pour votre enseignement, vos conseils et votre écoute tout au long de l'internat. Merci particulièrement à Damien pour ton aide précieuse pour mon mémoire. Merci également en particulier à Mme Popin pour votre disponibilité, votre écoute et votre enseignement.

A l'équipe de pneumologie de Saint-Quentin, en particulier à Youcef, pour le mémoire, et à Charles, Isabelle, Rémi, Manu, Houssine, Florence, pour tout ce que vous m'avez transmis, et dans la bonne humeur.

A l'équipe de Médecine Interne : Pr Duhaut, Dr Smail, Jean, Valéry, Florentina. Vous m'avez enseigné de nombreuses choses avec passion, mais j'ai encore l'espoir de vous convaincre que la pneumologie est la plus belle des spécialités !

$\mathrm{Au}$ service de Pneumologie de Saint-Joseph : Dr Salmeron, Claire, Jean-Luc, Christine, Pr Tredaniel, Marie, Stéphane, pour votre accueil et votre enseignement.

$\mathrm{Au}$ service de Pneumologie de Bichat: Pr Crestani, Camille, Raphaël, Marie-Christine, Clairelyne, Catherine, Mada, Pierre, pour la qualité de votre enseignement.

Merci à tous mes co-internes amiénois... On ne pouvait trouver mieux que vous deux pour cette promo de choc : Lola, ton éternelle fraîcheur et ton optimisme, Ugo, tes conversations et ta bienveillance, tous les deux sans limites ! Mickaël, le meilleur co-interne du monde (jusqu'à ce soir), merci pour tes conseils, ta bonne humeur, ton soutien (jusqu'à ce soir). Jérôme, mon premier co-interne et pas des moindres...ton humour nous manque! Alice, toujours partante pour toutes les aventures (sans râler ce serait encore mieux !). Florence, toujours première pour papoter (commérer)... Hortense, qui a joyeusement agrandi la famille amiénoise, Olivier, Marion, Marlène, Chloé et tous les autres avec qui je partage des souvenirs de stage ou de DES : Ozaire, Amel, Sarah, Nour, MAM, Pauline, Maxime, Warda, Fanny, Matthieu, Camille, Nathalie, Coco, Charlotte,...

Merci à Nouara pour ta bonne humeur... Merci aussi à Stéphanie Devaux, pour ton aide indispensable!

Merci à mes co-internes internistes, pour tous ces bons moments même si je ne vous comprends pas toujours: Caroline, dans son monde parallèle et Xavier, dans les hautes sphères de la médecine interne... Sans oublier l'extraordinaire Dr Brault, même si je n'ai toujours pas compris quelle était votre spécialité...

Merci à mes co-internes parisiens : Valentine, Domitille, Vincent, Hélène, Van et Louise, à bientôt au CPLF !

Merci à ma Cécile de Brouki, je suis arrivée là un peu grâce à toi et nos soirées studieuses (et nos soirées moins studieuses...). A Anne-Laurène, nos retrouvailles sont beaucoup trop rares! A tous les copains, de l'externat ou d'avant...merci pour les bons moments.

Merci à ma famille... Papa, pour tout ce que nous as transmis, le goût du travail, la persévérance. A Maman, pour ton courage et ta disponibilité. A Mathilde, Anne-Charlotte et Jérémy, qui supportez de ne m'entendre parler que de médecine... 


\section{Table des matières}

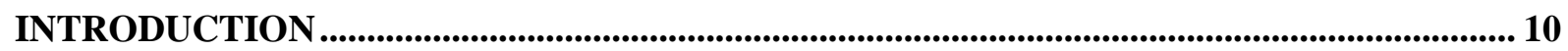

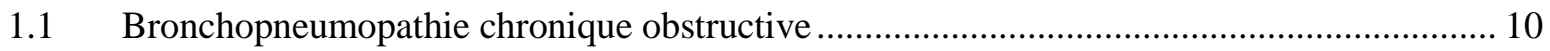

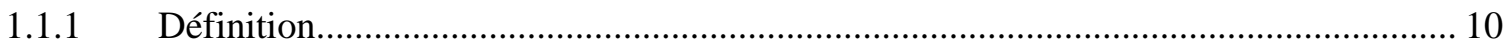

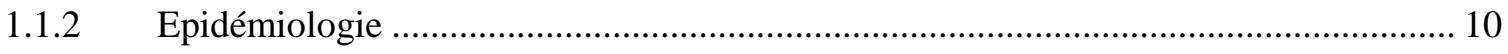

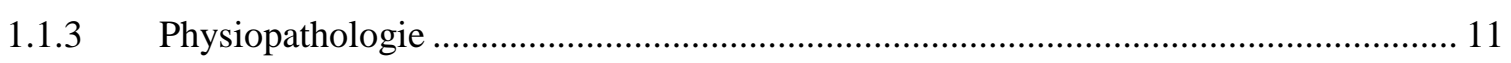

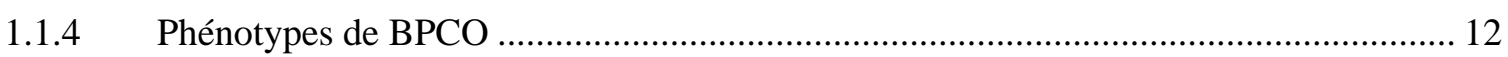

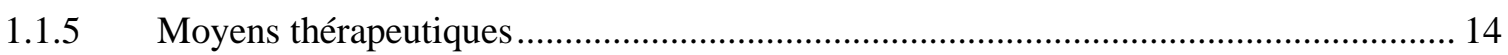

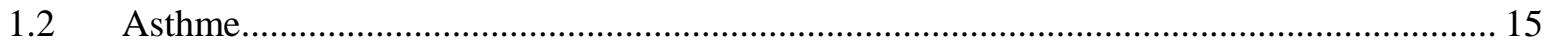

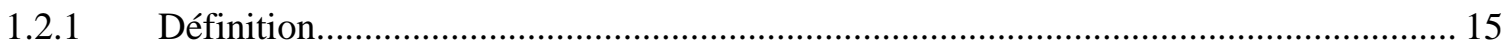

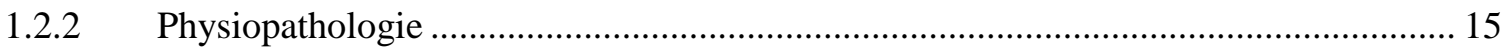

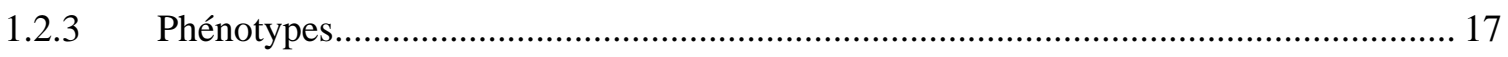

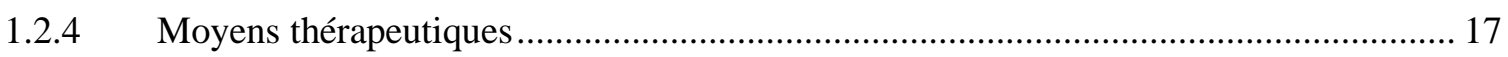

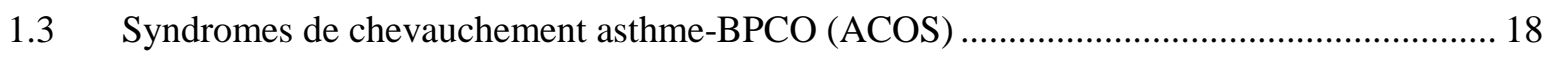

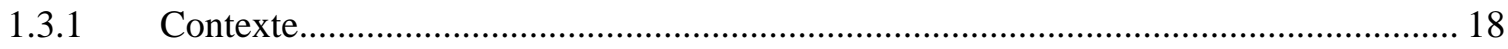

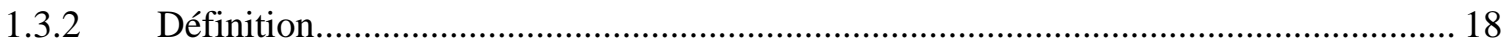

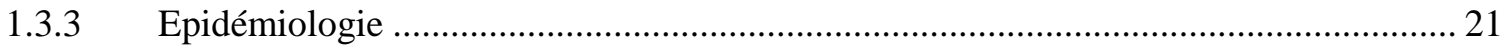

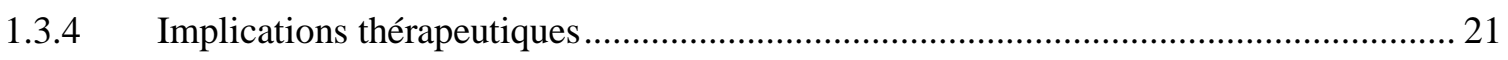

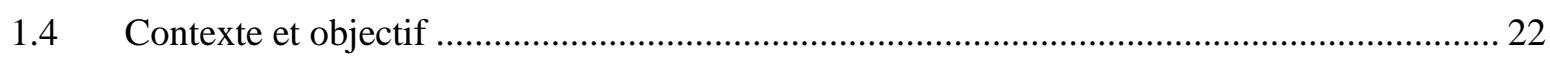

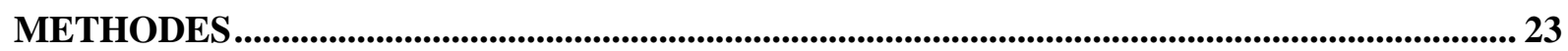

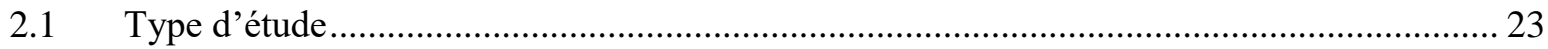

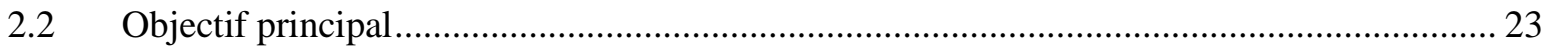

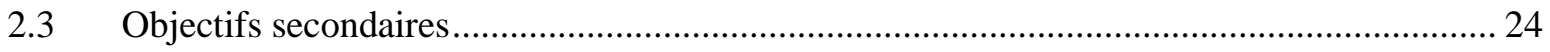

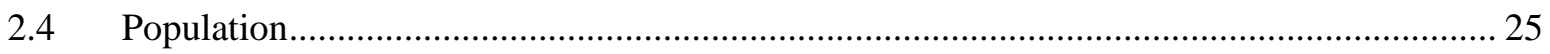

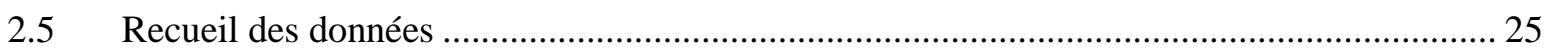

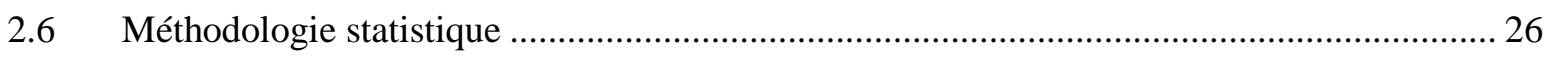

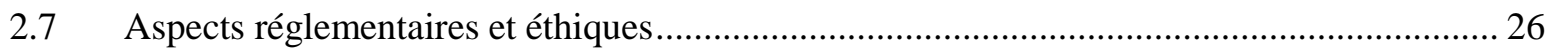

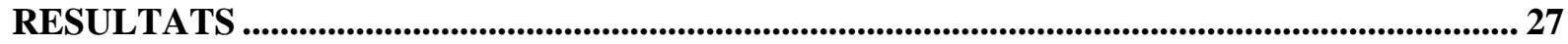

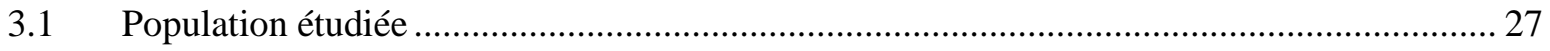

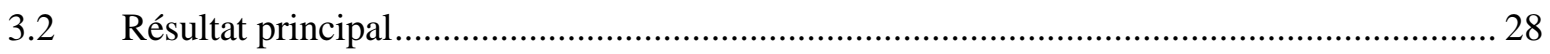

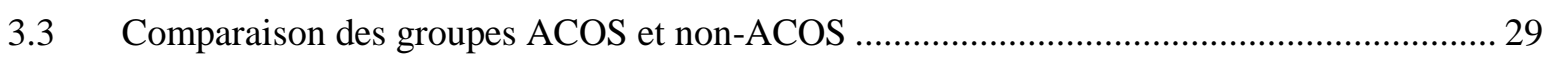

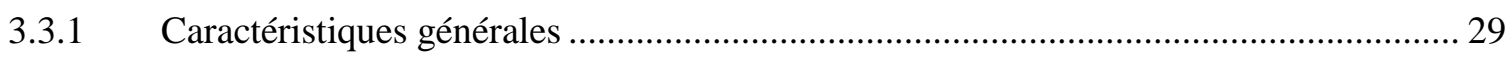

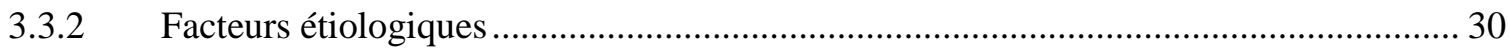

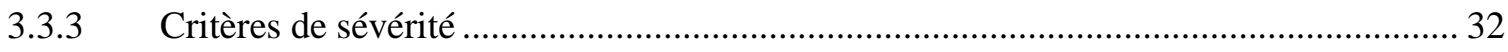

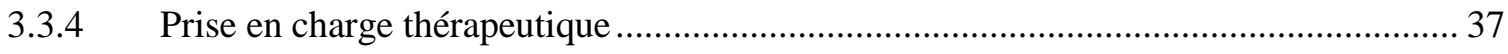

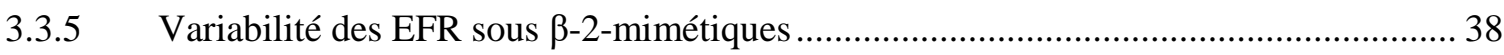




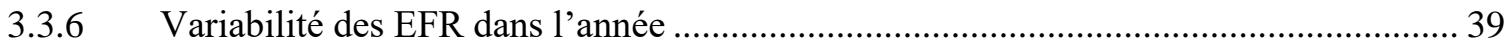

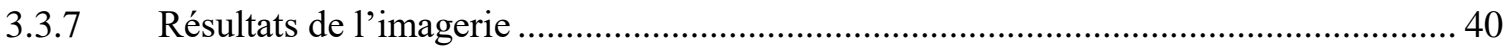

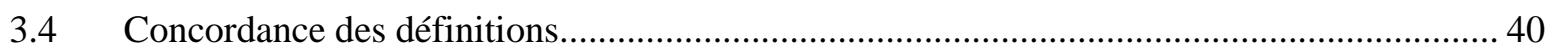

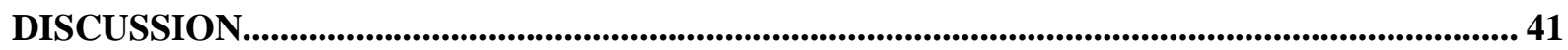

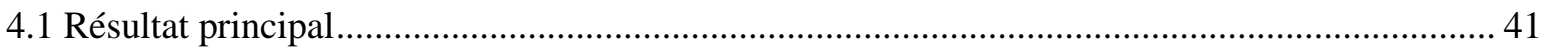

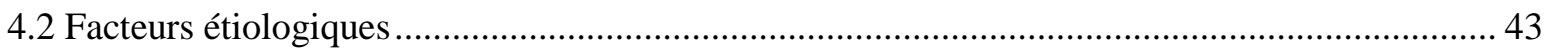

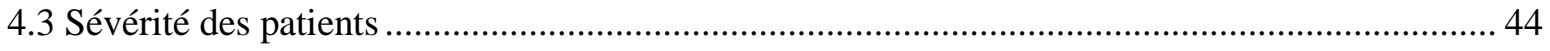

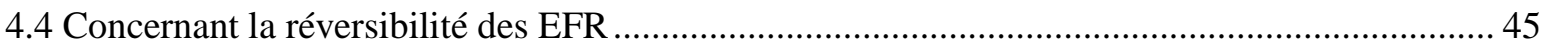

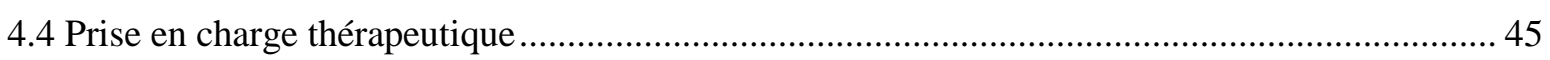

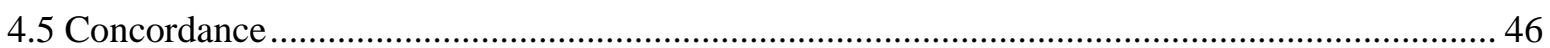

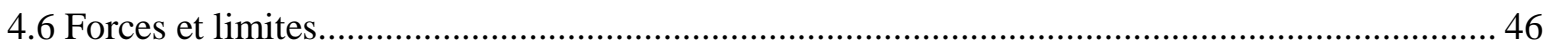

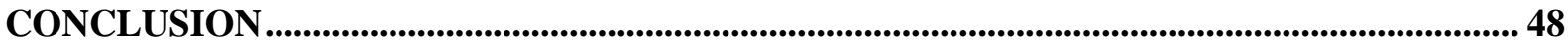

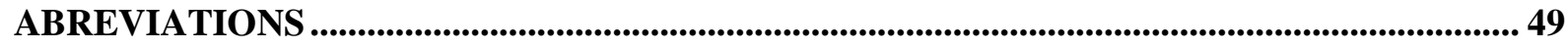

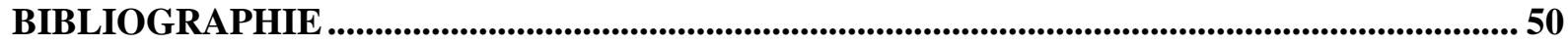




\section{INTRODUCTION}

\subsection{Bronchopneumopathie chronique obstructive}

\subsubsection{Définition}

La bronchopneumopathie chronique obstructive (BPCO) est une maladie bronchique inflammatoire chronique caractérisée par une obstruction bronchique persistante. Le diagnostic repose sur la mise en évidence d'un trouble ventilatoire obstructif (TVO) aux explorations fonctionnelles respiratoires (EFR) : rapport VEMS/CVF $<0,7$, de caractère fixé, c'est-à-dire sans réversibilité après test aux $\beta$-2-mimétiques. L'altération du VEMS définit le degré d'obstruction (1).

Les symptômes qui résultent de cette obstruction et qui doivent amener à pratiquer des EFR sont :

- $\quad$ Une dyspnée chronique et d'intensité croissante dans le temps.

- Une toux, pouvant s'accompagner d'une bronchorrhée claire en cas de bronchite chronique.

- Une distension thoracique

Cette définition implique que la toux chronique du fumeur et la bronchite chronique non associées à un trouble ventilatoire obstructif ne sont pas considérées comme des BPCO.

\subsubsection{Epidémiologie}

La BPCO est une maladie fréquente et chronique, ce qui entraîne une morbi-mortalité, un retentissement social et des coûts de santé importants.

Sa prévalence est difficile à estimer pour plusieurs raisons : il n'existe pas de registre officiel de BPCO, il existe un sous-diagnostic de la maladie (formes insidieuses ou prises en charge par le médecin traitant) et les études épidémiologiques avec mesures spirométriques sont difficiles à mettre en place. Les données actuelles reposent donc sur l'auto-déclaration et sous-estiment la prévalence réelle de la pathologie.

A l'échelle mondiale, on estime la prévalence de la BPCO à environ 384 millions de cas. La mortalité qui y est imputée est de 3 millions par an, ce qui la place comme $4^{\text {ème }}$ cause de mortalité mondiale $(2,3)$.

En France, la prévalence serait estimée à 7,5\% avec une mortalité supérieure à 16000 cas par an. Les exacerbations, qui représentent un poids considérable dans le coût de la maladie et la qualité de vie du patient, se compliquent fréquemment d'une hospitalisation. En 2007, on rapporte entre 69000 et 112000 hospitalisations pour exacerbation de BPCO (4). 
D'après l'Institut de Veille Sanitaire, les taux d'hospitalisation et de mortalité liés à la BPCO dans les Hauts-de-France seraient supérieurs de $20 \%$ à la moyenne nationale.

\subsubsection{Physiopathologie}

\subsubsection{Mécanismes immunologiques}

La physiopathologie de la BPCO n'est pas encore claire à ce jour. Si la BPCO a longtemps été considérée comme une maladie non inflammatoire, on reconnait actuellement de complexes voies inflammatoires.

Sur le plan cellulaire, l'inflammation neutrophilique est prédominante dans la plupart des $\mathrm{BPCO}$, avec une infiltration de la lumière bronchique et des expectorations à polynucléaires neutrophiles (5-7). Une corrélation a été observée entre l'inflammation locale de la paroi bronchique et le degré d'obstruction bronchique (8). La neutrophilie dans l'expectoration a aussi été identifiée comme un marqueur du degré d'obstruction et de sa progression et de la distension thoracique $(9,10)$. Il faut cependant pondérer ces résultats dans la mesure où la neutrophilie dans les expectorations augmente naturellement avec l'âge, et que seuls 34\% des BPCO auraient une neutrophilie significativement supérieure à celle des sujets sains (5).

Les lymphocytes CD8+ cytotoxiques et macrophages sont également au premier plan, infiltrant la sous-muqueuse bronchique de façon plus importante chez les BPCO que chez les bronchitiques chroniques sans trouble ventilatoire obstructif, et ce, particulièrement sur les bronches distales $(5,11)$. On retrouve également davantage de lymphocytes T CD8+ chez les patients BPCO fumeurs ou ex-fumeurs et une sévérité accrue de la BPCO en lien avec ces lymphocytes (5).

On constate cependant parfois une infiltration éosinophilique, définie par plus de 3\% d'éosinophiles dans l'expectoration. Ces patients présentent une réversibilité accrue aux $\beta-2-$ mimétiques (12), des taux de monoxyde d'azote exhalé plus élevés (13), et une plus grande amélioration du VEMS après traitement par corticoïdes (14). Ces patients présentent davantage de symptomatologie de bronchite chronique que les autres patients BPCO (15).

Sur le plan moléculaire, on observe une corrélation entre l'obstruction bronchique et la quantité d'IL-8 et de TNF, plus importante dans les expectorations de patients BPCO que dans celles de sujets sains ou de fumeurs non BPCO (16), témoignant d'une inflammation locale. La présence d'IL8 et de TNF semble être associée à un remodelage bronchique plus important, qui aboutit au trouble ventilatoire obstructif.

\subsubsection{Conséquences anatomopathologiques}

Le résultat de cette inflammation chronique est l'installation de lésions irréversibles. Ces lésions semblent siéger davantage sur les petites voies aériennes, entraînant une fibrose de la paroi des bronchioles terminales (5-7). 
L'étude anatomopathologique d'un poumon de patient BPCO révèle :

une fibrose des bronchioles : remodelage bronchique avec bronchoconstriction, qui se complique d'une diminution des débits expiratoires et de trapping.

- une destruction parenchymateuse : lésions d'emphysème, dues à un excès d'élastases et de protéases $(17,18)$

\subsubsection{Etiologie}

Les anomalies constatées dans la BPCO s'installent progressivement suite à l'interaction de facteurs environnementaux sur des terrains favorisants.

Concernant le terrain génétique, le déficit en alpha-1-antitrypsine, et particulièrement les formes $\mathrm{MZ}$ et $\mathrm{ZZ}$ sont associées à des formes précoces et graves de BPCO (19) mais ont l'avantage d'être accessibles à un traitement substitutif. D'autres anomalies génétiques, telles que des instabilités des microsatellites (20) ou des variations de gènes codant pour des protéases ont été identifiées sans pouvoir être accessibles à un traitement.

Concernant les facteurs environnementaux, le principal est le tabagisme, pour la première fois identifié comme facteur de risque dans la cohorte de Framingham.

Le second facteur de risque est l'exposition à la biomasse (fumée de feu de bois, exposition au charbon,...) qui représente jusqu'à $50 \%$ des causes de BPCO dans les pays en voie de développement (21), avec une surreprésentation des femmes à 75\% (22).

Les irritants professionnels pourraient représenter $15 \%$ des causes de BPCO selon l'American Thoracic Society (23) : en première place les expositions agricoles, céréalières ou animalières mais aussi les mineurs de charbon, les ouvriers du béton ou de la métallurgie (24).

La pollution atmosphérique semble aussi jouer un rôle, une étude britannique ayant montré une différence de prévalence de BPCO entre les facteurs travaillant en ville par rapport aux zones rurales (25).

Enfin certaines infections pourraient majorer le risque BPCO, sans qu'il n'y ait de preuve formelle. Une étude rétrospective menée en Afrique du Sud trouvait un risque de BPCO multiplié par 4 à 6 en cas de tuberculose en analyse multivariée, mais les données spirométriques n'étaient pas relevées et les symptômes décrits étaient ceux d'une bronchite chronique (26).

\subsubsection{Phénotypes de BPCO}

L'identification de phénotypes parmi les patients BPCO est une idée ancienne, évoquée depuis l'établissement de la définition de la BPCO dans les années 1960, devant le constat de tableaux cliniques différents.

Plusieurs tentatives de classification ont été proposées, se basant sur différents critères. 
En considérant les caractéristiques cliniques ont été établis les phénotypes de «blue bloater » ayant une bronchite chronique, une surcharge pondérale, une hypoxémie précoce et de «pink puffer » caractérisés par une dyspnée importante sans bronchite chronique, un emphysème important avec une distension thoracique et une dénutrition marquée.

Si l'on considère des caractéristiques fonctionnelles, la classification du groupe Global initiative for chronic Obstructive Lung Disease (GOLD) s'est longtemps basée sur le degré d'obstruction, traduit par le VEMS, reflétant davantage la sévérité de la maladie que de véritables phénotypes. L'étude ECLIPSE a d'ailleurs rendu cette classification insuffisante en montrant que, pour un même degré d'obstruction, la dyspnée, le retentissement sur les activités quotidiennes et le nombre d'exacerbations ne sont pas identiques (27).

Une autre classification a ensuite été proposée par le groupe GOLD (fig.1), donnant 4 groupes associés à un risque d'exacerbation différent, se basant sur le degré d'obstruction et sur la dyspnée selon l'échelle mMRC et un score de qualité de vie (score CAT) (1).

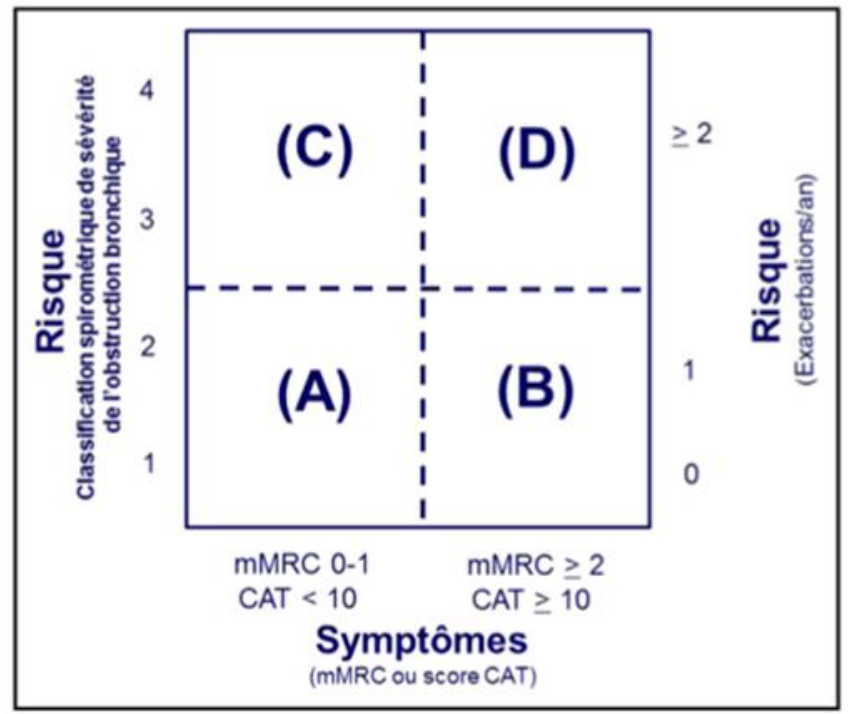

Figure 1 : Classification Gold 2017

Les travaux actuels tendent à identifier des profils de BPCO définis par leur mécanisme physiopathologique et immunologique, sans y parvenir de façon satisfaisante, notamment en l'absence de biomarqueur existant pour caractériser la BPCO.

Néanmoins, des ébauches ont été évoquées. Des formes de BPCO avec hyperéosinophilie dans les expectorations ont été décrites. Ces formes semblent présenter une symptomatologie de bronchite chronique, une réversibilité et une réponse aux corticoïdes inhalés plus importantes que dans les formes sans hyperéosinophilie $(14,28,29)$. Ces caractéristiques en font des formes proches de l'asthme.

A l'inverse, les BPCO avec inflammation neutrophilique semblent être associées à des images scanographiques de distension thoracique et à une obstruction plus importantes (9). 


\subsubsection{Moyens thérapeutiques}

L'objectif du traitement de la BPCO est de diminuer les symptômes et de limiter la progression de la maladie, notamment en prévenant les exacerbations.

Outre les mesures hygiéno-diététiques et en premier lieu le sevrage tabagique et l'activité physique, le groupe GOLD recommande une prise en charge pharmacologique adaptée au stade de BPCO telle que décrite dans la figure 2 (1) :
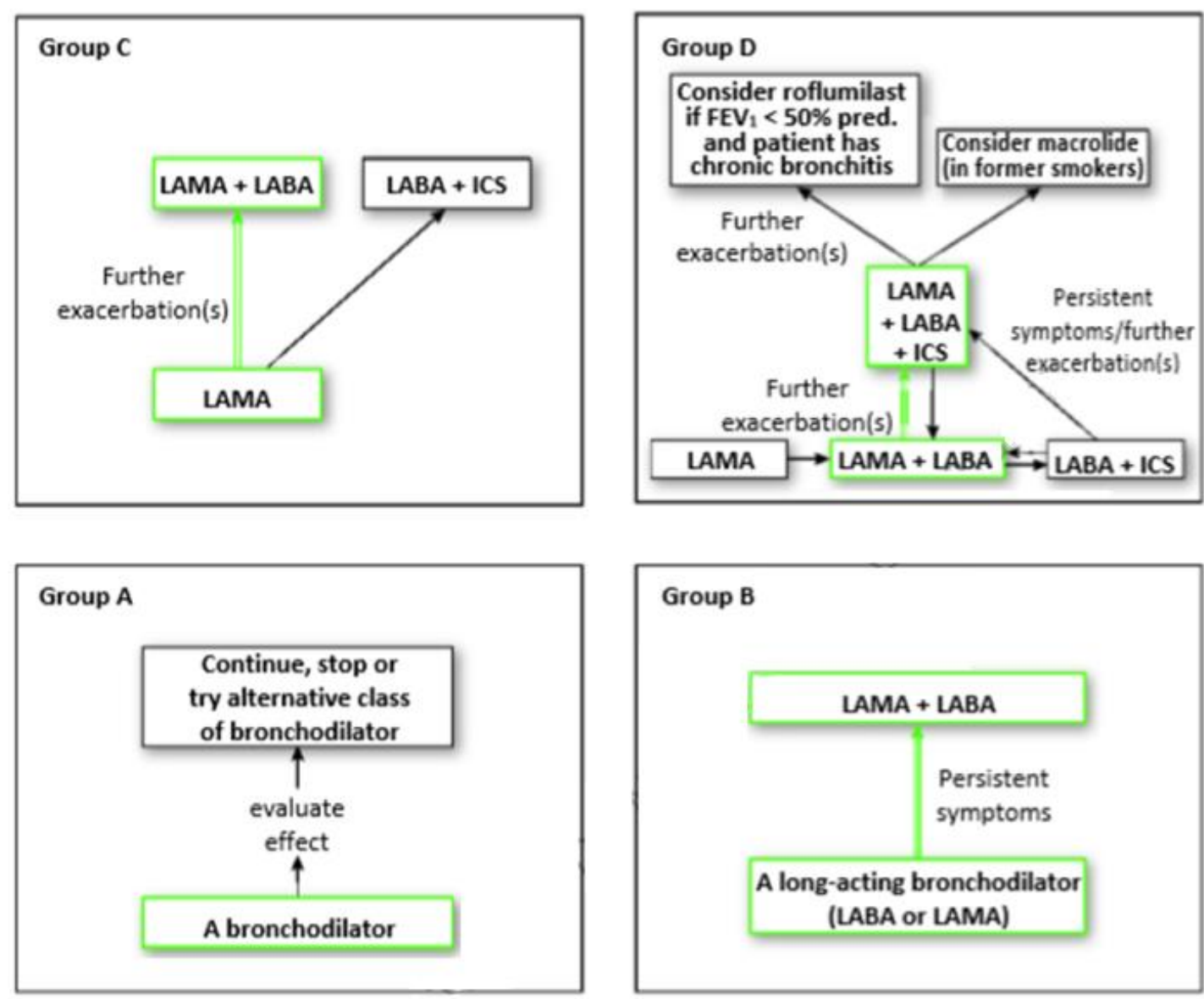

Group B

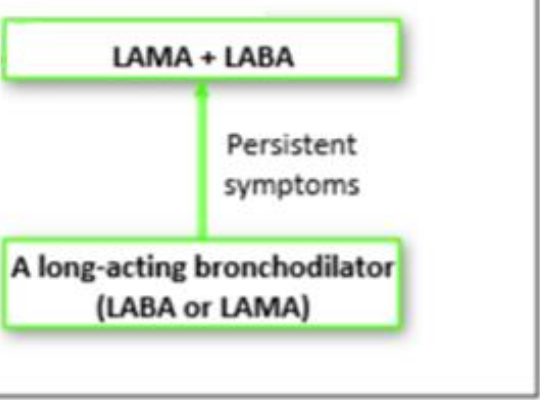

Figure 2 : Recommandations de prise en charge de la BPCO selon le groupe GOLD. LAMA :bronchodilatateurs muscariniques (anticholinergiques) de longue durée d'action, $L A B A$ :bronchodilatateurs $\beta$-2-mimétiques de longue durée d'action, ICS :corticostérö̈des inhalés.

On constate que les principaux traitements sont les bronchodilatateurs de longue durée d'action, anti-cholinergiques et/ou $\beta$-2-mimétiques.

La place des corticostéroïdes inhalés (CSI) est donc limitée. En effet, dans la mesure où l'inflammation est surtout neutrophilique, la réponse aux corticoïdes inhalés est faible. Seuls certains patients sont éligibles à un traitement par CSI ; il s'agit des exacerbateurs fréquents, chez qui une diminution de la fréquence des exacerbations de $25 \%$ et une amélioration de la qualité de vie sous corticoïdes inhalés a été montrée (28). 
Par ailleurs, une plus grande réponse aux CSI a été démontrée chez les patients présentant une forte hyperréactivité bronchique ou une augmentation du NO exhalé, qui sont des caractéristiques habituellement rattachées à l'asthme. De même une éosinophilie dans les expectorations, ou une éosinophilie sanguine, reflet indirect des expectorations, ont montré une augmentation de la réponse aux CSI chez les BPCO $(14,28,29)$.

En revanche, chez les BPCO ne répondant pas à ces critères, il n'existe pas d'effet démontré des CSI (7) mais plutôt une majoration du risque de complications à type de colonisation par des mycobactéries et pneumopathies infectieuses. Ils majorent aussi le risque d'ostéoporose, de pneumopathie et de candidose oropharyngée ainsi que les coûts de santé (6).

Les CSI semblent donc n'avoir leur place, en cas de BPCO, qu'en cas d'exacerbations fréquentes ou de forme frontière et leur prescription doit être limitée à ces patients.

\subsection{Asthme}

\subsubsection{Définition}

L'asthme est également une maladie inflammatoire chronique des bronches, mais qui se manifeste de façon ponctuelle contrairement à la BPCO. Le trouble ventilatoire obstructif (VEMS/CVF < 0,7) est en effet réversible sous $\beta$-2-mimétiques (gain de VEMS supérieur à $200 \mathrm{~mL}$ et $12 \%)$.

Les symptômes sont donc paroxystiques, à type de sifflements expiratoires, de dyspnée et de toux dont l'existence et l'intensité varient en fonction du degré d'obstruction. Leur survenue est variable dans le temps, pouvant être absente pendant des mois voire des années, spontanée ou secondaire à un facteur favorisant.

\subsubsection{Physiopathologie}

\subsubsection{Etiologie}

Le plus souvent, l'asthme survient dans un contexte familial, déclenché par des allergènes et associé à l'atopie.

En dehors de ce contexte allergique, on trouve des asthmes intrinsèques, déclenchés par des irritants respiratoires, ou s'intégrant dans d'autres tableaux pathologiques tels que des vascularites, nettement plus rares.

\subsubsection{Mécanismes immunologiques}

Le schéma suivant (fig.3) résume les acteurs impliqués dans la réaction asthmatique (30). 


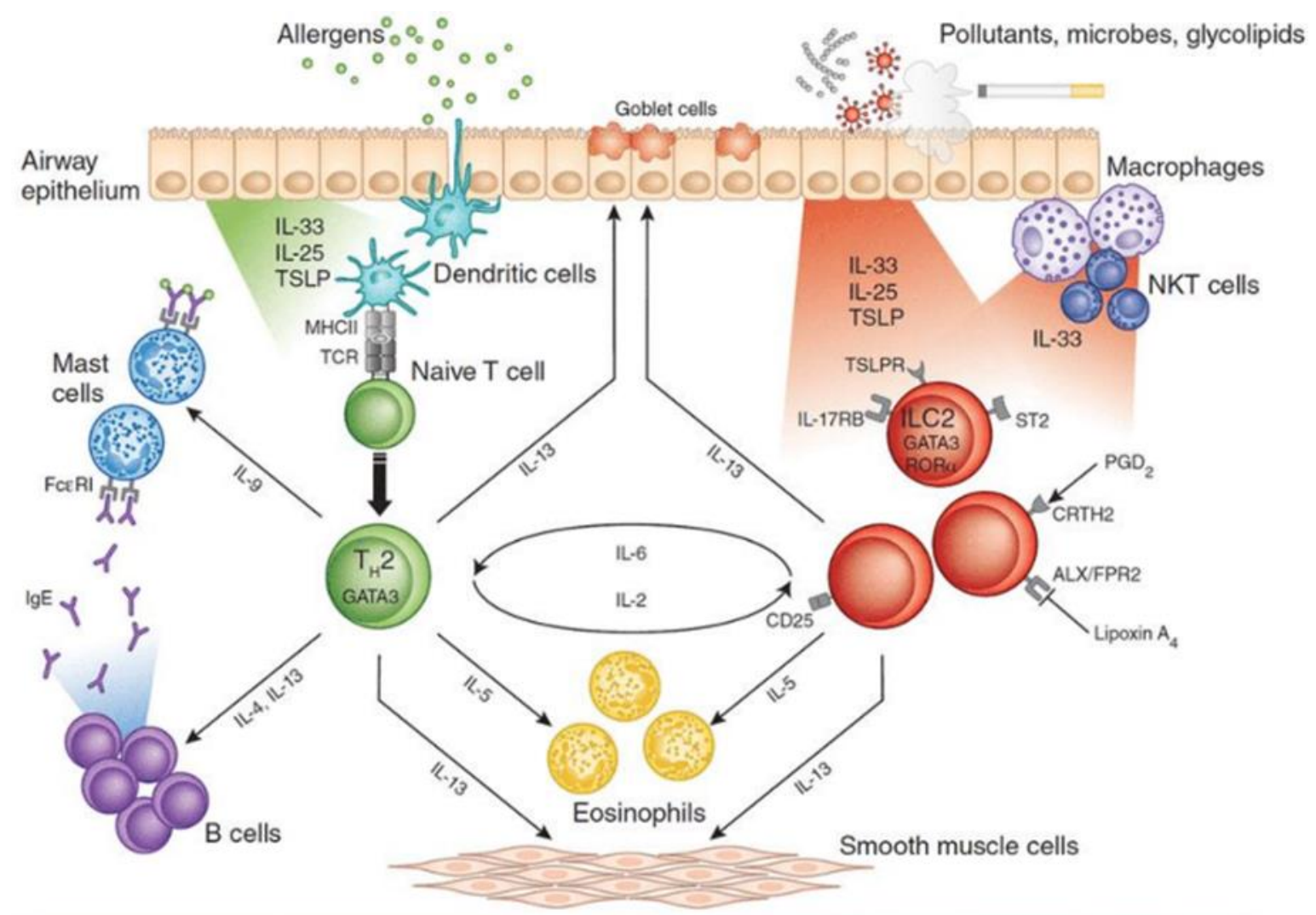

Allergic eosinophilic airway inflammation Nonallergic eosinophilic airway inflammation

Figure 3 : Voies inflammatoires impliquées dans l'asthme

La voie la mieux connue et la plus fréquemment impliquée est la voie Th2, activée par des stimuli allergéniques, via des mécanismes d'hypersensibilité immédiate impliquant les IgE (31).

L'autre voie est activée par des irritants respiratoires et n'implique pas les lymphocytes Th2, mais ces deux voies ne sont pas complètement indépendantes l'une de l'autre, interagissant via des interleukines sur les éosinophiles.

Sur le plan cellulaire, les éosinophiles et les mastocytes sont au premier plan, activés par les lymphocytes T CD4 via la voie Th2. On note qu'en cas d'asthme non allergique, l'éosinophile garde un rôle primordial (31).

Sur le plan moléculaire, les cytokines libérées par les Th2, IL-5 et IL-13, vont activer les éosinophiles et vont donc pouvoir être la cible de thérapeutiques contre l'asthme. L'IL-4 et l'IL-13, sécrétées par le lymphocyte $\mathrm{T}$, activent les lymphocytes $\mathrm{B}$ qui vont conduire à la synthèse d'IgE, et peuvent également être la cible de traitements d'asthmes sévères. 


\subsubsection{Conséquences anatomopathologiques}

Deux composantes sont impliquées dans l'asthme :

- $\quad$ Une composante inflammatoire, responsable d'une hypersécrétion bronchique.

- L'hyperréactivité bronchique, secondaire à une hypertrophie des cellules musculaires lisses, responsable d'une bronchoconstriction réversible.

L'hyperréactivité bronchique dans l'asthme peut être sévère et est plus importante que dans la BPCO. Elle est corrélée à l'épaisseur des parois bronchiques et du muscle lisse et à la fermeture des voies aériennes sous métacholine (10). Dans l'asthme, cette hypertrophie des muscles lisses prédomine sur les bronches proximales.

\subsubsection{Phénotypes}

En raison d'une hétérogénéité des tableaux d'asthme, des tentatives de classification ont été menées, afin d'identifier différents phénotypes d'asthme.

Ces classifications peuvent se baser sur l'aspect clinique : obésité, âge, sévérité des symptômes, ou sur l'aspect biologique : inflammation éosinophilique ou neutrophilique. Il n'existe pas de classification reconnue par l'ensemble des scientifiques, mais les données sont convergentes pour distinguer (32):

- Les asthmes éosinophiliques, intégrant l'asthme allergique, l'asthme tardif de l'adulte, non atopique, et l'asthme avec intolérance à l'aspirine.

- Les asthmes neutrophiliques, comprenant l'asthme du fumeur et l'asthme de l'obèse

\section{$\underline{1.2 .4}$ Moyens thérapeutiques}

Le traitement de l'asthme est variable suivant le niveau de contrôle de l'asthme. Au minimum, en cas d'asthme contrôlé, sera utilisé un bronchodilatateur à la demande de type $\beta$ 2-mimétique d'action rapide (32).

En cas de symptômes persistants, le traitement reposera sur les corticoïdes inhalés, qui seront, en fonction du contrôle de la maladie, majorés en posologie et/ou associés à des bronchodilatateurs d'action lente. Les corticoïdes semblent présenter un effet très limités dans les asthmes neutrophiliques (32).

Depuis la connaissance des mécanismes immunologiques se sont également développées des biothérapies, ciblant les IgE, tel quel l'Omalizumab, ou 1'IL-5, tel que le Mépolizumab. 


\subsection{Syndromes de chevauchement asthme-BPCO (ACOS)}

\subsubsection{Contexte}

L'asthme et la BPCO ont longtemps été vus comme deux entités strictement distinctes, et il peut être facile de poser le diagnostic en cas de présentation typique. Pourtant, certains patients présentent un trouble ventilatoire obstructif qu'il est difficile de rattacher à l'une ou à l'autre de ces maladies de façon certaine, dans la mesure où ils présentent des caractéristiques de l'une et de l'autre. Pour ces formes, dites de chevauchement, a été suggérée la notion d'Asthma-and-COPD-overlap syndrome (ACOS) dans le début des années 2000, terme qui regroupe un ensemble de troubles ventilatoires obstructifs se rapprochant de la BPCO et/ou de l'asthme. Depuis 2017, il est recommandé de plutôt utiliser le terme d'ACO pour insister sur le fait qu'il s'agit de différentes entités et non pas d'une maladie indépendante (32).

Devant ces constatations, un diagramme de Venn est proposé (fig.4), suggérant un continuum entre l'asthme, la BPCO et l'emphysème, avec des formes de chevauchement.

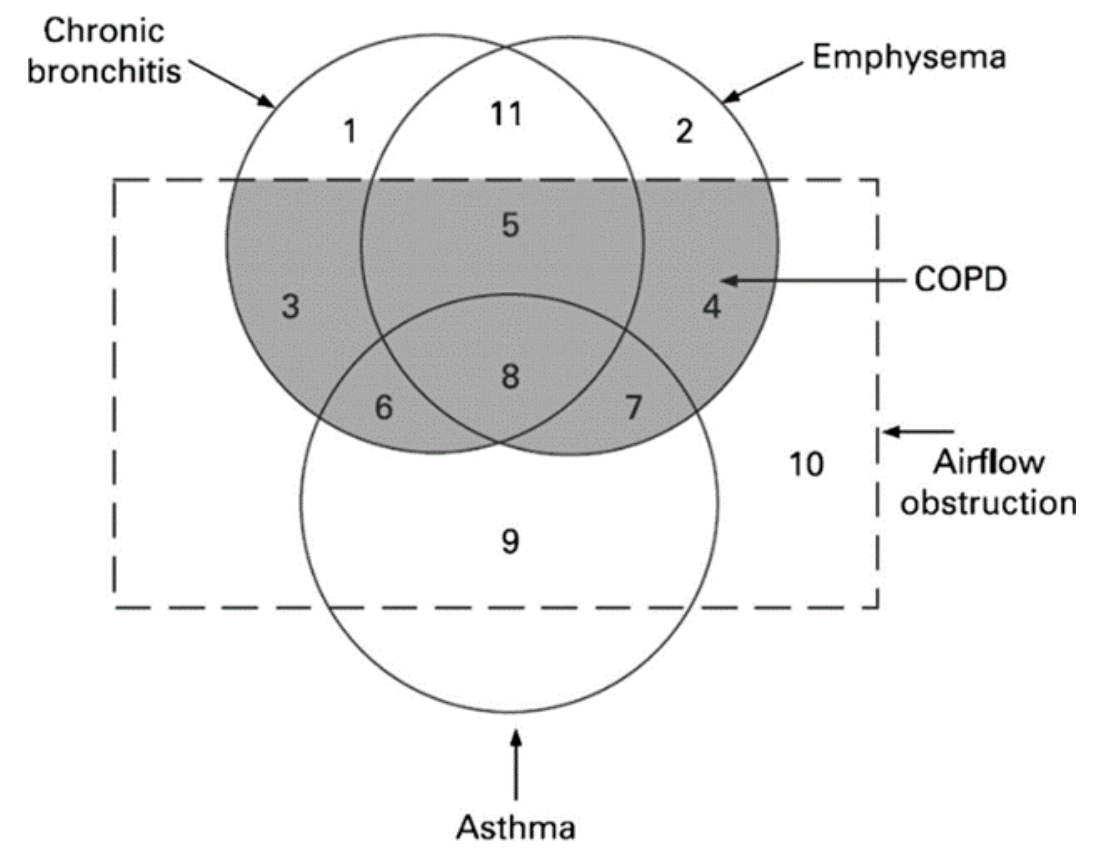

Figure 4 : Diagramme de Venn des bronchopathies chroniques. Les ACOS correspondent aux aires 6, 7, 8 .

\subsubsection{Définition}

Actuellement, il n'existe pas de définition stricte pour définir l'ACOS en raison de l'hétérogénéité des formes englobées par cet acronyme. De nombreuses études ont tenté de proposer une définition (33). 
En 2014, le Global INitiative for Asthma (GINA), et le GOLD se sont penchés sur la question afin de proposer une définition consensuelle. Ce qu'ils proposent est plutôt une description qu'une définition de l'ACOS, désigné « trouble ventilatoire obstructif persistant associé à des caractéristiques usuellement rattachées à l'asthme ou à la BPCO » (32).

Ils ont pour cela établi une liste de ces caractéristiques :

- $\quad$ Age de début des symptômes : avant ou après 40 ans, respectivement plus en faveur de l'asthme ou de la BPCO.

- Variabilité des symptômes : variation horaire ou persistance dans le temps, respectivement plus en faveur de l'asthme ou de la BPCO.

- Variabilité de la fonction respiratoire : réversibilité du trouble ventilatoire obstructif ou stabilité, respectivement plus en faveur de l'asthme ou de la BPCO.

- $\quad$ Fonction respiratoire entre les symptômes : normale ou persistance d'altération des EFR, respectivement plus en faveur de l'asthme ou de la BPCO.

- $\quad$ Antécédents personnels ou familiaux d'asthme ou d'atopie, plus en faveur de l'asthme.

- Evolution dans le temps : amélioration après des périodes d'aggravation ou lente aggravation, respectivement plus en faveur de l'asthme ou de la BPCO.

- $\quad$ Critères radiologiques : distension thoracique, en faveur de la BPCO.

Les diagnostics d'asthme ou de BPCO seraient retenus si 3 critères ou plus étaient en faveur de l'un ou de l'autre et le diagnostic d'ACO si le même nombre de critères appartenant à l'asthme et à la BPCO était retrouvé (fig.5). 


\begin{tabular}{|c|c|c|c|c|c|}
\hline Feature & Asthma & COPD & $\begin{array}{c}\text { Asthma-COPD } \\
\text { overlap }\end{array}$ & $\begin{array}{c}\text { More likely to be asthma } \\
\text { if several of ...n }\end{array}$ & $\begin{array}{c}\text { More likely to be COPD } \\
\text { if several of...* }\end{array}$ \\
\hline ge of onset & $\begin{array}{l}\text { Usually childhood onset } \\
\text { but can commence at any } \\
\text { age. }\end{array}$ & Usually $>40$ years of age & $\begin{array}{l}\text { Usually age } 240 \text { years, but may } \\
\text { have had symptoms in } \\
\text { childhood or early adulthood }\end{array}$ & $\square$ Onset before age 20 years & $\square$ Onset after age 40 years \\
\hline $\begin{array}{l}\text { Pottern of } \\
\text { respiratory } \\
\text { symptoms }\end{array}$ & $\begin{array}{l}\text { Symptoms may vary over } \\
\text { time (day to day, or over } \\
\text { longer periods), often } \\
\text { limiting activity. Often } \\
\text { triggered by exercise, } \\
\text { emotions including } \\
\text { laughter, dust or } \\
\text { exposure to allergens }\end{array}$ & $\begin{array}{l}\text { Chronic usually continuous } \\
\text { symptoms, particularly } \\
\text { during exercise, with } \\
\text { 'better' and 'worse' days }\end{array}$ & $\begin{array}{l}\text { Respiratory symptoms including } \\
\text { exertional dyspnea are } \\
\text { persistent but variability may } \\
\text { be prominent }\end{array}$ & $\begin{array}{l}\text { Variation in symptoms over } \\
\text { minutes, hours or days } \\
\text { symptoms worse during the } \\
\text { night or earlymorning } \\
\text { o symptoms triggered by exercise, } \\
\text { emotions including laughter, } \\
\text { dust or exposure to allergens }\end{array}$ & $\begin{array}{l}\square \text { Persistence of symptoms despit } \\
\text { treatment } \\
\square \text { Good and bad days but ahways } \\
\text { daily symptoms and exertional } \\
\text { dyspnea } \\
\square \text { Chronic cough and sputum } \\
\text { preceded onset of dyspnea, } \\
\text { unrelated to triggers }\end{array}$ \\
\hline Lung function & $\begin{array}{l}\text { Current and/or historical } \\
\text { variable airflow limitation, } \\
\text { e.g. BD reversibility, AHR }\end{array}$ & $\begin{array}{l}\text { FEV }, \text { may be improved by } \\
\text { therapy, but post-BD } \\
\mathrm{FEV}_{2} / \mathrm{FVC}<0.7 \text { persists }\end{array}$ & $\begin{array}{l}\text { Airflow limitation not fully } \\
\text { reversible, but often with } \\
\text { current or historical variability }\end{array}$ & $\begin{array}{l}\text { D Record of variable airflow } \\
\text { limitation (spirometry, peak } \\
\text { flow) }\end{array}$ & $\begin{array}{l}\text { D Record of persistent airflow } \\
\text { limitation (post-bronchodilator } \\
\mathrm{FEV}_{2} / \mathrm{FVC}<0.7 \text { ) }\end{array}$ \\
\hline $\begin{array}{l}\text { Lung function } \\
\text { between } \\
\text { symptoms }\end{array}$ & $\begin{array}{l}\text { May be normal between } \\
\text { symptoms }\end{array}$ & Persistent airflow limitation & Persistent airflow limitation & $\begin{array}{l}\text { L tung function normal between } \\
\text { symptoms }\end{array}$ & $\begin{array}{l}\text { L Lung function abnormal } \\
\text { between symptoms }\end{array}$ \\
\hline $\begin{array}{l}\text { Post history } \\
\text { or fomily } \\
\text { history }\end{array}$ & $\begin{array}{l}\text { Many patients have } \\
\text { allergies and a personal } \\
\text { history of asthma in } \\
\text { childhood, and/or family } \\
\text { history of asthma }\end{array}$ & $\begin{array}{l}\text { History of exposure to } \\
\text { noxious particles and gases } \\
\text { (mainly tobacco smoking } \\
\text { and biomass fuels) }\end{array}$ & $\begin{array}{l}\text { Frequently a history of doctor- } \\
\text { diagnosed asthma (current or } \\
\text { previous), allergies and a family } \\
\text { history of asthma, and/or a } \\
\text { history of noxious exposures }\end{array}$ & $\begin{array}{l}\text { Previous doctor diagnosis of } \\
\text { asthma } \\
\text { Family history of asthma, and } \\
\text { other allergic conditions (allergic } \\
\text { rhinitis or eczema) }\end{array}$ & $\begin{array}{l}\square \text { Previous doctor diagnosis of } \\
\text { COPD, chronic bronchitis or } \\
\text { emphysema } \\
\square \text { Heary exposure to a risk factor: } \\
\text { tobacco smoke, biomass fuels }\end{array}$ \\
\hline Time course & $\begin{array}{l}\text { Often improves } \\
\text { spontaneously or with } \\
\text { treatment, but may result } \\
\text { in foxed airflow limitation }\end{array}$ & $\begin{array}{l}\text { Generally, slowly } \\
\text { progressive over year } \\
\text { despite treatment }\end{array}$ & $\begin{array}{l}\text { symptoms are partly but } \\
\text { significantly reduced by } \\
\text { treatment. Progression is usual } \\
\text { and treatment needs are high }\end{array}$ & $\begin{array}{l}\text { No worsening of symptoms over } \\
\text { time. Symptoms vary either } \\
\text { seasonally, or from year to year } \\
\square \text { May improve spontaneously or } \\
\text { have an immediate response to } \\
\text { BD or to ICS over weeks }\end{array}$ & $\begin{array}{l}\text { Symptoms slowly worsening } \\
\text { over time (progressive course } \\
\text { over years) } \\
\text { Rapid-acting bronchodilator } \\
\text { treatment provides only limited } \\
\text { relief. }\end{array}$ \\
\hline Chest $x$-roy & Usually normal & $\begin{array}{l}\text { Severe hvperinflation \& } \\
\text { other changes of COPD }\end{array}$ & Similar to COPD & D Normal & D severe hyperinflation \\
\hline Exacerbations & $\begin{array}{l}\text { Exacerbations occur, but } \\
\text { the risk of exacerbations } \\
\text { can be considerably } \\
\text { reduced by treatment }\end{array}$ & $\begin{array}{l}\text { Exacerbations can be } \\
\text { reduced by treatment. If } \\
\text { present, comorbidities } \\
\text { contribute to impairment }\end{array}$ & $\begin{array}{l}\text { Exacerbations may be more } \\
\text { common than in copo but are } \\
\text { reduced by treatment. } \\
\text { comorbidities can contribute to } \\
\text { impairment }\end{array}$ & \multirow{2}{*}{\multicolumn{2}{|c|}{$\begin{array}{l}\text { *Syndromic diagnosis of airways disease: how to use Box 5-2b } \\
\text { Shaded columns list feotures that, when present, best identify potients } \\
\text { with typical osthmo ond COPD. For a potient, count the number of } \\
\text { check boxes in eoch column. If three or more boxes ore checked for } \\
\text { either osthma or COPD, the potient is likely to hove thot disease. If } \\
\text { there ore similor numbers of checked boxes in each column, the } \\
\text { diagnosis of ACO should be considered. See Step } 2 \text { for more details. }\end{array}$}} \\
\hline $\begin{array}{l}\text { Airwoy } \\
\text { inflommotion }\end{array}$ & $\begin{array}{l}\text { Eosinophils and/or } \\
\text { neutrophils }\end{array}$ & $\begin{array}{l}\text { Neutrophils } \pm \text { eosinophils in } \\
\text { sputum, lymphocytes in } \\
\text { ainways, may have systemic }\end{array}$ & $\begin{array}{l}\text { Eosinophils and/or neutrophils } \\
\text { in sputum. }\end{array}$ & & \\
\hline
\end{tabular}

Figure 5 : Distinction des asthmes, BPCO et ACOS selon le GINA

Cette définition ne portant pas sur des critères stricts, un groupe d'experts s'est réuni en 2016 afin de proposer un consensus (34). Leur définition requiert 3 critères majeurs et un mineur :

Parmi les critères majeurs :

- une obstruction bronchique persistante après bronchodilatateurs (rapport VEMS/CVF < $70 \%$ ) chez un sujet de plus de 40 ans.

- un tabagisme supérieur à 10 paquets-années (PA) OU une exposition environnementale à la biomasse

- une histoire documentée d'asthme avant l'âge de 40 ans OU une amélioration du VEMS supérieure à $400 \mathrm{~mL}$ après bronchodilatateurs

Parmi les critères mineurs :

- histoire documentée d'atopie ou de rhinite allergique

- amélioration du VEMS > 200mL et $12 \%$ de la valeur de base après bronchodilatateurs, évaluée lors de 2 consultations ou plus.

- éosinophilie sanguine $>300 / \mathrm{mm} 3$ 


\subsubsection{Epidémiologie}

La prévalence des ACOS parmi les BPCO est difficile à estimer du fait qu'il s'agisse d'une notion récente, pour laquelle peu d'études épidémiologiques ont été conduites, et de l'absence de définition précise, rendant les comparaisons difficiles. On estime que $6,5 \%$ à $55 \%$ des patients BPCO pourraient présenter un ACOS (35-38), l'étude qui retrouve un taux de 6,5\% se basant sur des critères restrictifs, imposant une réversibilité supérieure à $400 \mathrm{~mL}$. L'étude qui retrouve un taux à 55\% (35) se base sur des critères de réversibilité assez larges tels que la variabilité du Peak-flow ou l'existence d'un wheezing, que l'on peut constater dans la BPCO, et pourrait surestimer le nombre d'ACOS. Les deux autres études citées trouvaient des taux entre 13 et $17 \%$ sans donner une définition stricte d'ACOS ( « caractéristiques d'asthme et de $\mathrm{BPCO} \gg)$.

L'ACOS pourrait, du fait de sa définition large, être considéré comme un phénotype de BPCO. Ces patients semblent en effet différents des autres patients BPCO, plus jeunes et avec une proportion de femmes plus importante (39) et un tabagisme moins important.

Ils présentent d'avantage de signes évocateurs d'atopie : rhinite allergique, hypersensibilité avec élévation des IgE ou positivité des pricks tests (40-42). D'un point de vue physiopathologique, on constate une inflammation plutôt éosinophilique, avec une élévation du taux de NO exhalé se rapprochant donc plutôt de l'asthme que de la BPCO avec une réversibilité plus importante aux EFR $(13,43)$. Quant à l'imagerie, les ACOS présentent davantage d'épaississements bronchiques et moins d'emphysème que les BPCO (44).

Concernant la gravité des ACOS, les données sont divergentes. Plusieurs études ont montré un risque d'hospitalisation plus important (45). Une étude multicentrique mais sans définition précise de l'ACOS montrait un risque d'hospitalisation 4 fois plus important, d'exacerbation 2 fois plus important et une qualité de vie moindre en cas d'ACOS par rapport à une BPCO (38) et des coûts de santé plus importants en raison d'une consommation médicamenteuse plus importante et de recours plus fréquents au médecin traitant et à l'hospitalisation $(39,46)$. D'autres études montrent à l'inverse une meilleure survie des phénotypes ACOS par rapport aux BPCO exacerbateurs fréquents ou emphysémateux $(43,47)$.

\subsubsection{Implications thérapeutiques}

L'identification de ces formes de chevauchement permet d'une part de mieux comprendre les mécanismes physiopathologiques impliqués dans ces maladies, et d'autre part d'adapter la thérapeutique.

L'adaptation thérapeutique reste à ce jour minime pour plusieurs raisons. L'arrivée récente de cette notion n'a pas permis le développement de nombreux essais thérapeutiques. Par ailleurs, les classiques études portant sur l'asthme et la BPCO ont des critères d'inclusion très sélectifs, excluant donc toute forme de chevauchement. Par exemple, les patients tabagiques sont classiquement exclus des essais faits chez les patients asthmatiques. Bien qu'attendue, l'efficacité des CSI dans les ACOS n'a donc pas été prouvée à ce jour. 
Néanmoins, des essais ont démontré une efficacité de certains traitements développés pour l'asthme dans la prise en charge de BPCO : le mépolizumab, anticorps anti IL-5, a montré son efficacité sur la réduction des exacerbations chez les BPCO présentant un profil éosinophilique (48) et l'omalizumab, anticorps anti-IgE, a montré son efficacité sur la réduction des exacerbations et du nombre d'hospitalisations (49).

Il semble donc intéressant de s'intéresser au sous-groupe de patients BPCO présentant un syndrome de chevauchement.

\subsection{Contexte et objectif}

La BPCO est en Picardie un problème de santé publique en raison de sa prévalence et de sa surmortalité. Il semble donc légitime d'étudier les caractéristiques de ces patients BPCO. L'émergence de la notion d'ACOS fait se poser la question de ces phénotypes parmi les patients BPCO de Picardie.

Nous nous sommes attachés à évaluer la prévalence des patients répondants aux critères $\mathrm{d}^{\prime} \mathrm{ACOS}$ et à leurs caractéristiques parmi les patients suivis pour une BPCO au CHU d'Amiens. 


\subsection{Type d'étude}

Il s'agit d'une étude épidémiologique monocentrique observationnelle descriptive rétrospective portant sur la cohorte des patients BPCO du CHU d'AMIENS sur la période de mai 2017 à mai 2018.

\subsection{Objectif principal}

L'objectif principal de l'étude était d'estimer la prévalence des patients pouvant répondre aux critères d'ACOS parmi les patients suivis pour une BPCO au CHU d'AMIENS.

La principale difficulté était de définir ces ACOS, dans la mesure où il n'existe pas de définition consensuelle. Il a donc été décidé d'utiliser les deux définitions citées dans l'introduction.

La première était un score diagnostique (fig.6) basé sur le tableau du GINA, les arguments en faveur d'une BPCO comptant pour un point positif, les arguments en faveur d'un asthme pour un point négatif. Le diagnostic d'ACOS était retenu en présence d'un score compris entre -2 et 2 inclus. Cette définition (50), bien qu'elle n'ait pas été validée par d'autres études, a l'avantage de se baser sur les recommandations GINA et GOLD.

La seconde définition était issue d'un consensus d'expert (34). Le diagnostic était retenu en présence de 3 critères majeurs :

- une obstruction bronchique persistante après bronchodilatateurs (rapport VEMS/CVF < $70 \%$ ) chez un sujet de plus de 40 ans.

- un tabagisme supérieur à 10 paquets-années (PA) ou une exposition environnementale.

- une histoire documentée d'asthme avant l'âge de 40 ans OU une amélioration du VEMS supérieure à $400 \mathrm{~mL}$ après bronchodilatateurs.

Et un critère mineur parmi :

- une histoire documentée d'atopie ou de rhinite allergique

- une amélioration du VEMS > 200mL et > $12 \%$ de la valeur de base après bronchodilatateurs, évaluée lors de 2 consultations ou plus.

- une éosinophilie sanguine > 300/mm3 


\begin{tabular}{|c|c|c|c|c|}
\hline & En taveur d'un ASTHME & Points & En faveur d'une BPCO & Points \\
\hline \multirow[t]{4}{*}{ Symptômes } & Débutent avant räge de 20 ans & -1 & $\square$ Débutent après ràge de $\mathbf{4 0}$ ans & +1 \\
\hline & $\begin{array}{l}\text { Variation en quelques minutes, heures ou } \\
\text { jours }\end{array}$ & -1 & $\square$ Persistent malgré le traitement & +1 \\
\hline & $\square$ Pires la nuit ou au petit matin & -1 & $\begin{array}{l}\text { a * Bons * et \& mauvais * jours mais } \\
\text { symptómes quotidiens et dyspnée d'effort } \\
\text { toujours présente }\end{array}$ & +1 \\
\hline & $\begin{array}{l}\text { Déclenchés par rexercice physique, les } \\
\text { émotions, rexposition aux poussières ou aux } \\
\text { allergènes }\end{array}$ & -1 & $\begin{array}{l}\text { Une toux chronique et des } \\
\text { expectorations ont précédé rapparition } \\
\text { de la dyspnée, pas de facteur } \\
\text { déclenchant }\end{array}$ & +1 \\
\hline \multirow{2}{*}{$\begin{array}{l}\text { Antécédents } \\
\text { personnels ou } \\
\text { familiaux }\end{array}$} & - Diagnostic d'asthme dans le passé & -1 & $\begin{array}{l}\text { Diagnostic de BPCO, de bronchite } \\
\text { chronique ou demphysème dans le passé }\end{array}$ & +1 \\
\hline & $\begin{array}{l}\text { Anamnèse familiale d'asthme, dreczéma ou } \\
\text { d'allergies }\end{array}$ & -1 & $\begin{array}{l}\text { Exposition importante à un tacteur de } \\
\text { risque (tabagisme, fumée de biomasse) }\end{array}$ & +1 \\
\hline \multirow[t]{2}{*}{ Evolution } & $\begin{array}{l}\text { Pas daggravation avec le temps. Variation } \\
\text { saisonnière ou d'une annee sur I'autre }\end{array}$ & -1 & $\begin{array}{l}\text { Aggravation lentement progressive avec } \\
\text { les annees }\end{array}$ & +1 \\
\hline & $\begin{array}{l}\text { Peut s'améliorer spontanément. Amélioration } \\
\text { rapide aprés BD ou en quelques semaines avec } \\
\text { les CSI }\end{array}$ & -1 & $\begin{array}{l}\text { Les BD à courte durée d'action } \\
\text { n'apportent qu'une amélioration modérée }\end{array}$ & +1 \\
\hline \multirow[t]{3}{*}{$\begin{array}{l}\text { Radiographie du } \\
\text { thorax }\end{array}$} & - Normale & -1 & Hyperinflation plus ou moins sévère & +1 \\
\hline & Points négatifs & & Points positifs & \\
\hline & \multicolumn{4}{|c|}{ Asthme possible ACOS $\leq-3 \quad \geq+3$} \\
\hline
\end{tabular}

Figure 6: Score diagnostique

\subsection{Objectifs secondaires}

Les objectifs secondaires étaient de comparer les caractéristiques des patients admis dans le groupe ACOS par rapport aux autres patients BPCO non emphysémateux afin de rechercher des différences en termes de caractéristiques phénotypiques, de sévérité et de caractéristiques spirométriques.

Il a également été décidé d'évaluer la concordance diagnostique entre les deux tests. 


\subsection{Population}

Nous nous sommes intéressés aux patients ayant consulté pour une BPCO au CHU d'Amiens sur la période de mai 2017 à mai 2018. La population éligible était déterminée après lecture du courrier de consultation. Tout patient pour lequel le diagnostic de BPCO était indiqué par un pneumologue pouvait être inclus.

Après recueil de cette liste, étaient secondairement exclus :

- les autres pathologies bronchiques, pouvant représenter un facteur confondant : aspergilloses bronchopulmonaires allergiques, mucoviscidose, dilatations des bronches localisées ou diffuses. N'étaient pas exclus les épaississements de parois bronchiques diffus fréquemment vus dans l'asthme et la BPCO.

- $\quad$ Les emphysèmes (autres que centro-lobulaires minimes qui étaient tolérés), évocateur d'un autre phénotype de BPCO.

- Les patients présentant un diagnostic différentiel associé (pneumopathie interstitielle diffuse, cancer bronchopulmonaire, syndrome restrictif) en raison d'anomalies fonctionnelles et cliniques associées à ces pathologies.

- $\quad$ Les patients n'ayant réalisé qu'une EFR sans test de réversibilité sur l'année, en raison de l'impossibilité d'évaluer la variabilité des EFR

\subsection{Recueil des données}

Les données ont été recueillies de façon rétrospective, par étude du dossier médical : recueil des données des courriers de consultation et d'hospitalisation, lecture des EFR, lecture de l'imagerie.

Les données recueillies étaient d'ordre :

démographique : date de naissance, sexe, poids, taille.

environnemental : tabagisme actif ou passif, quantification du tabagisme, exposition professionnelle à des agents susceptibles de provoquer un asthme, exposition professionnelle à des agents susceptibles de provoquer une BPCO, exposition domestique à des allergènes ou irritants.

- $\quad$ médical : âge des premiers symptômes, antécédents personnel d'asthme diagnostiqué par un médecin dans l'enfance, antécédents d'allergies certaines, alimentaires, respiratoires ou médicamenteuses, atopie (rhinite, eczéma), antécédents familiaux d'asthme ou d'allergie ou atopie.

fonctionnel : dyspnée sur l'échelle mMRC, existence d'une bronchite chronique, données des EFR : VEMS/CVF, VEMS à l'état stable, test de marche de 6 mn (TM6), nombre d'exacerbations, de consultations ou d'hospitalisations par an et facteur déclenchant. 
- thérapeutique : corticoïdes oraux ou inhalées, $\beta$-2-mimétiques d'action rapide, anticholinergiques, montelukast, omalizumab, mépolizumab, Anti-histaminiques, azithromycine.

- $\quad$ pronostique : indice BODE, mortalité.

- biologique : éosinophiles sanguins, IgE totales, IgE aspergillaires, IgG aspergillaires, éosinophiles dans les expectorations $(>2,5 \%)$, Aspergillus dans les expectorations

- $\quad$ gazométrique : $\mathrm{pO}_{2}$ et $\mathrm{pCO}_{2}$

- $\quad$ radiologique et scanographique

\subsection{Méthodologie statistique}

Une analyse de la population a d'abord été réalisée, avec présentation des données qualitatives en fréquence et pourcentage et des données quantitatives en moyenne et écart type ou médianes et extrêmes selon la distribution. Une analyse bivariée a ensuite été réalisée pour comparer (selon les deux définitions d'ACOS) les patients ayant un ACOS de ceux ayant une BPCO sans ACOS avec pour les variables qualitatives un test du Chi2 ou un test exact de Fisher et pour les variables quantitatives une comparaison de moyenne selon la méthode de Student ou un test non paramétrique de Wilcoxon selon la distribution. La corrélation entre les tests a été évaluée par un test Rho de Spearman. Un p inférieur à 0,5 a été considéré comme significatif. Les analyses ont été faites grâce au logiciel SPSS 12.0.

\subsection{Aspects réglementaires et éthiques}

Compte tenu du caractère rétrospectif et monocentrique, il n'y a pas eu de CPP. Une déclaration a été faite à la direction du CHU d'Amiens, ainsi qu'à la CNIL. 


\subsection{Population étudiée}

L'étude a été réalisée à partir de la cohorte de patients ayant consulté en pneumologie au CHU d'Amiens entre le 15 mai 2017 et le 15 mai 2018.

Le screening a retenu les patients venus pour «bronchite chronique » ou «BPCO», délimitant 495 patients.

Parmi les critères d'exclusion se trouvaient les autres atteintes pulmonaires, interstitielles ou bronchiques, ou des pathologies lourdes telles que l'insuffisance cardiaque terminale ou les patients dialysés, dont la pathologie de fond pouvait retentir sur la symptomatologie respiratoire ou les EFR.

Après exclusion des patients présentant une pathologie associée ou un manque de données, on recensait 369 patients avec un diagnostic de BPCO dans le courrier, dont 203 avec un phénotype d'emphysème. Il existait donc 166 patients présentant une BPCO nonemphysémateuse. Cependant, après l'inclusion des 166 patients, nous avons relevé 32 patients dont le rapport VEMS/CVF était supérieur à 0,7 et ne répondaient donc pas à la définition actuelle de la BPCO.

Parmi ces 32 patients, se trouvaient :

- 5 patients suivis pour bronchite chronique

- 11 patients qualifiés de BPCO par abus de langage, présentant une bronchite chronique sans trouble ventilatoire obstructif associé

- 5 patients qualifiés de BPCO en raison d'un rapport VEMS/CVF limite $(0,71-0,72)$ et d'une symptomatologie compatible

- 9 patients qualifiés de BPCO car présentant un rapport VEMS/CV inférieur à 0,7 , mais un rapport VEMS/CVF $>0,7$

- 2 patients qui avaient eu un rapport VEMS/CVF inférieur à 0,7 constaté à une seule reprise antérieurement, et dont les EFR sur la période de recueil étaient normalisées.

La prévalence des ACOS a été évaluée sur l'ensemble des patients ayant un diagnostic exclusif de BPCO et pour les patients ayant réellement un trouble ventilatoire obstructif. Les analyses comparatives ont en revanche été réalisées sur la population de BPCO qui présentait réellement un trouble ventilatoire obstructif (fig.7). 


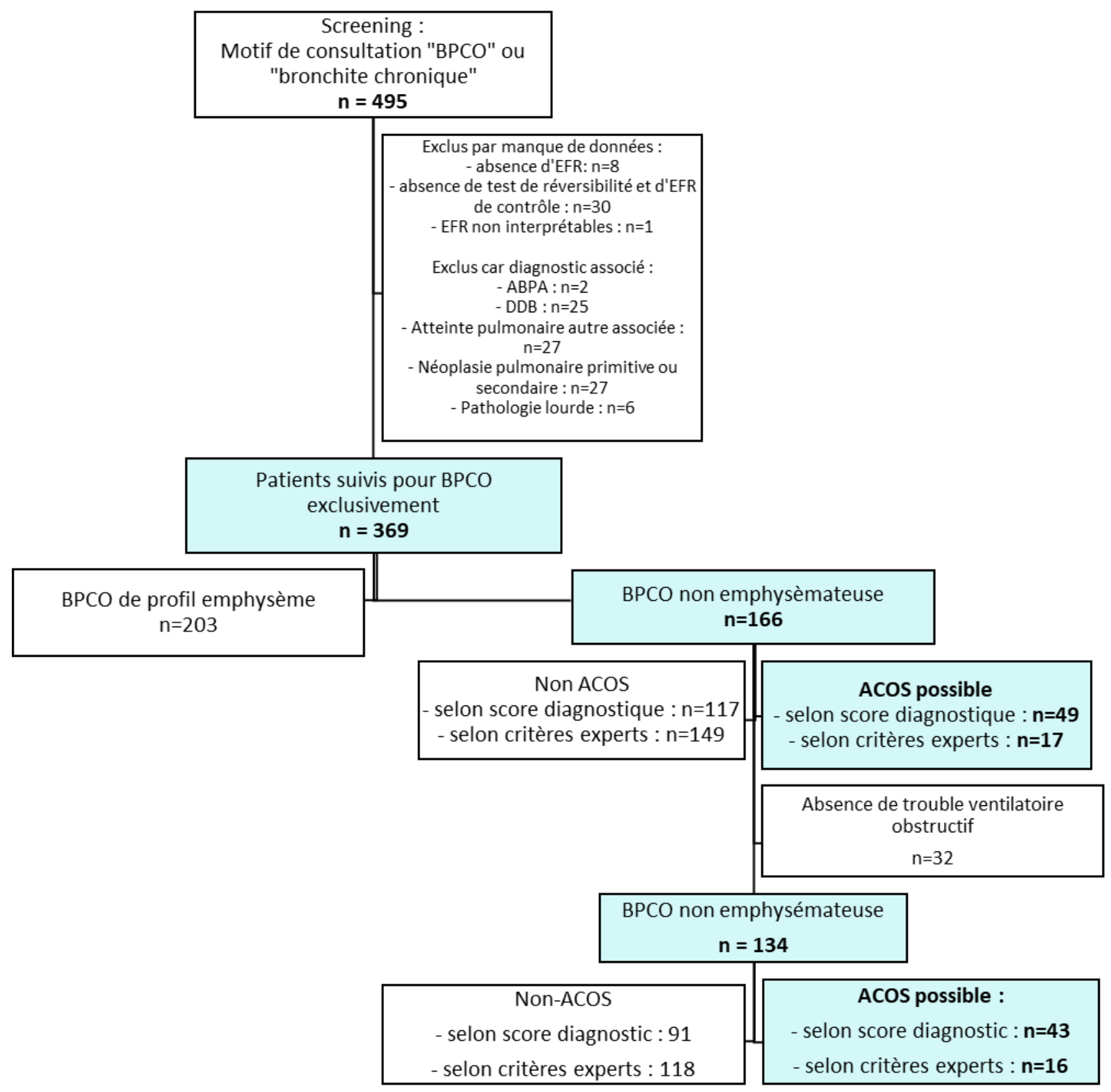

Figure 7 : Répartition de la cohorte de BPCO du CHU d'Amiens

\subsection{Résultat principal}

L'objectif principal était d'évaluer la prévalence des patients pouvant répondre aux critères d'ACOS parmi les patients suivis pour BPCO au CHU d'Amiens, en se basant sur deux définitions.

Si l'on considère la population globale des patients suivis pour un diagnostic exclusif de BPCO (phénotypes emphysémateux et non-emphysémateux compris), on observe une 
prévalence de 13,28\% selon la définition du score diagnostique, et de 4,61\% selon la définition issue du consensus d'experts (fig.8).

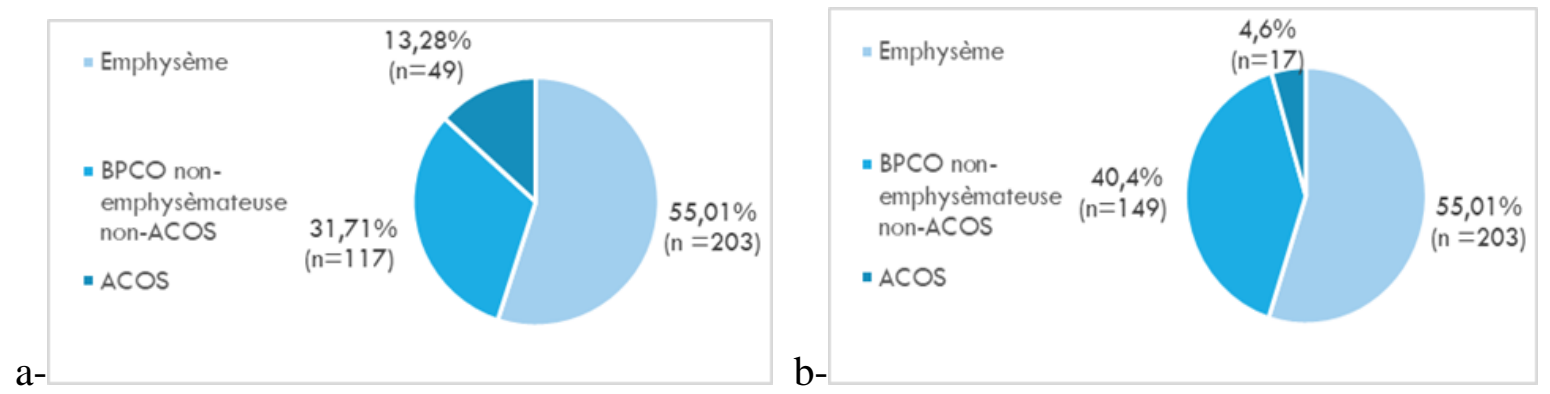

Figure 8: Prévalence des ACOS parmi les patients BPCO du CHU d'Amiens selon le score diagnostique (a) ou la définition du consensus d'experts (b)

Si l'on restreint aux patients BPCO non-emphysémateux (166 patients), 29,5\% correspondent à un ACOS selon le score diagnostique, et 10,2\% selon la définition issue du consensus d'experts.

Si l'on excluait les 32 patients qui présentaient un rapport VEMS/CVF supérieur à $70 \%, 43$ patients sur 134 soit 32,1\% des patients étaient des ACOS possibles selon le score diagnostique, 16 patients sur 134 soit 11,2\% étaient des ACOS possibles selon la définition issue du consensus (fig.9).
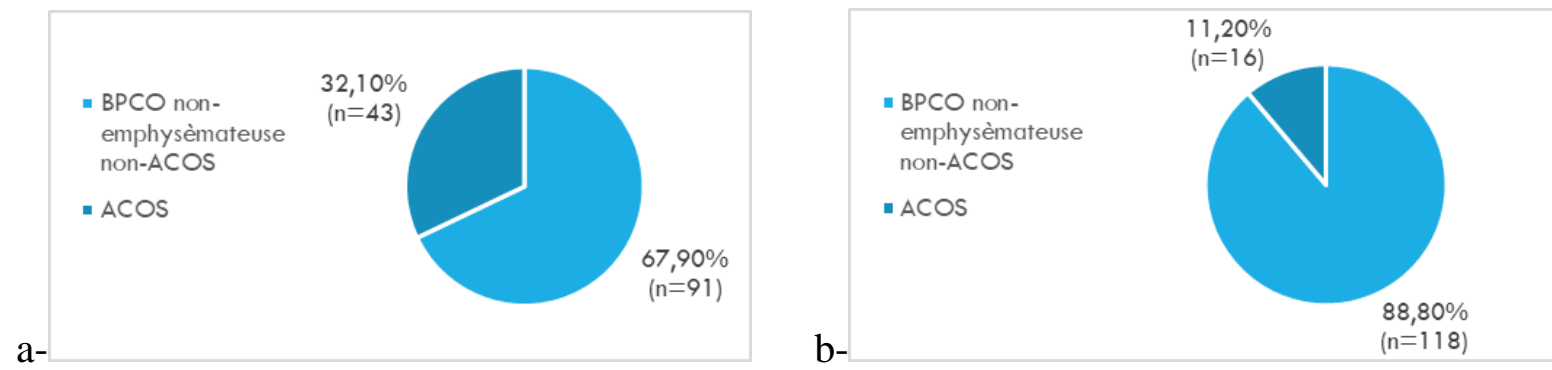

Figure 9 : Prévalence des ACOS parmi les patients BPCO du CHU d'Amiens selon le score diagnostique (a) ou la définition $d u$ consensus d'experts (b) après exclusion des emphysémateux et des rapports VEMS/CVF >0,7.

\subsection{Comparaison des groupes ACOS et non-ACOS}

\subsubsection{Caractéristiques générales}

Les études comparatives ont porté sur les 134 patients BPCO chez qui le trouble ventilatoire obstructif avait été confirmé (exclusion des patients avec un rapport VEMS/CVF > 0,7).

Il existait dans cette population une surreprésentation des hommes, deux fois plus présents que les femmes. L'âge moyen était de 67 ans au moment de l'inclusion. Le rapport VEMS/CVF moyen était de $60 \%$, avec des extrêmes entre $36 \%$ et $70 \%$. Une bronchite chronique était rapportée pour $42,5 \%$ des patients. 
Il n'a pas été mis en évidence de différence entre les groupes ACOS et non-ACOS en termes de sexe, d'âge, d'IMC ou de symptomatologie bronchitique quelle que soit la définition utilisée pour définir les ACOS (Tableaux 1 et 2).

\begin{tabular}{lllll}
\hline & $\begin{array}{l}\text { Population générale } \\
(\mathrm{n}=134)\end{array}$ & $\begin{array}{l}\text { Non-ACOS } \\
(\mathrm{n}=91)\end{array}$ & $\begin{array}{l}\text { ACOS } \\
(\mathrm{n}=43)\end{array}$ & $\mathrm{p}$ \\
\hline Sexe masculin & $90(67,2 \%)$ & $62(68,1 \%)$ & $28(65,1 \%)$ & 0,844 \\
Age (années) & $67,06 \pm 10,83$ & $67,9 \pm 10,13$ & $66,5 \pm 12,81$ & 0,50 \\
IMC (kg/m2) & $29,9 \pm 6,24$ & $29,9 \pm 6,67$ & $29,8 \pm 5,27$ & 0,93 \\
Rapport VEMS/CVF & $60,0 \% \pm 0,08$ & $59,9 \% \pm 8,3$ & $60,1 \% \pm 6,3$ & 0,920 \\
Bronchite chronique & $57(42,5 \%)$ & $40(44,0 \%)$ & $17(39,5 \%)$ & 0,710 \\
\hline
\end{tabular}

Tableau 1 : Caractéristiques générales de la population en fonction de l'existence d'un ACOS selon le score diagnostique.

\begin{tabular}{lllll}
\hline & $\begin{array}{l}\text { Population générale } \\
(\mathrm{n}=134)\end{array}$ & $\begin{array}{l}\text { Non-ACOS } \\
(\mathrm{n}=118)\end{array}$ & $\begin{array}{l}\text { ACOS } \\
(\mathrm{n}=16)\end{array}$ & $\mathrm{p}$ \\
\hline Sexe masculin & $90(67,2 \%)$ & $80(67,8 \%)$ & $10(62,5 \%)$ & 0,778 \\
Age (années) & $67 \pm 10,83$ & $67,9 \pm 10,61$ & $64,1 \pm 13,70$ & 0,197 \\
IMC (kg/m2) & $29,9 \pm 6,24$ & $29,7 \pm 6,39$ & $31,2 \pm 4,99$ & 0,367 \\
Rapport VEMS/CVF & $60,0 \% \pm 0,08$ & $59,7 \pm 7,71$ & $61,9 \% \pm 7,24$ & 0,282 \\
Bronchite chronique & $57(42,5 \%)$ & $51(43,2 \%)$ & $6(37,5 \%)$ & 0,790 \\
\hline
\end{tabular}

Tableau 2 : Caractéristiques générales de la population en fonction de l'existence d'un ACOS selon le consensus d'experts.

\subsubsection{Facteurs étiologiques}

Les facteurs reconnus comme inducteurs de BPCO, dont le tabagisme et certaines expositions professionnelles, étaient renseignés respectivement chez $100 \%$ et 85,8\% des 134 patients.

Les facteurs impliqués dans l'asthme, antécédents d'allergie ou d'asthme, antécédents familiaux d'asthme ou d'atopie, exposition professionnelle, étaient renseignés chez respectivement $99,3 \%, 97,8 \%, 79,1 \%, 78,4 \%$ et $86,6 \%$ des 134 patients.

Quel que soit la définition utilisée, aucune différence n'était constatée entre les deux groupes concernant le tabagisme.

En utilisant la définition issue du score diagnostique, on trouvait une différence significative de variabilité des symptômes dans la journée, d'antécédents personnels d'asthme ou d'antécédents familiaux d'asthme ou d'atopie, de déclenchement des symptômes par les allergènes, plus importants dans le groupe ACOS que dans le groupe non-ACOS. Ceci était cohérent dans la mesure où ces critères étaient inclus dans le score diagnostique.

L'éosinophilie en revanche, non comprise dans le score, était également plus élevée dans le groupe ACOS de façon significative (Tableau 3). 


\begin{tabular}{|c|c|c|c|c|}
\hline & $\begin{array}{l}\text { Population } \\
\text { générale } \\
(\mathrm{n}=134)\end{array}$ & $\begin{array}{l}\text { Non-ACOS } \\
(\mathrm{n}=91)\end{array}$ & $\operatorname{ACOS}(n=43)$ & $\mathrm{p}$ \\
\hline Age de début des symptômes (années) & $49,64 \pm 19,9$ & $56,8 \pm 15,33$ & $34,11 \pm 19,91$ & $<0,001$ \\
\hline $\begin{array}{l}\text { Variabilité des symptômes dans la } \\
\text { journée }\end{array}$ & $14(10,4 \%)$ & $3(3,3 \%)$ & $11(25,6 \%)$ & $<0,001$ \\
\hline Antécédent d'allergie ou d'atopie & $18(13,4 \%)$ & $9(9,9 \%)$ & $9(20,9 \%)$ & 0,177 \\
\hline Antécédent d'asthme & $37(27,6 \%)$ & $8(8,8 \%)$ & $29(67,4 \%)$ & $<0,001$ \\
\hline Antécédents familiaux d'asthme & $8(6,0 \%)$ & $3(3,3 \%)$ & $5(11,6 \%)$ & 0,047 \\
\hline Antécédents familiaux d'atopie & $2(1,5 \%)$ & $0(0 \%)$ & $2(4,7 \%)$ & $\mathbf{0 , 0 2 3}$ \\
\hline $\begin{array}{l}\text { Exposition professionnelle à des } \\
\text { facteurs inducteurs d'asthme }\end{array}$ & $21(15,7 \%)$ & $13(14,3 \%)$ & $8(18,6 \%)$ & 0,631 \\
\hline Tabagisme actif & $126(94,0 \%)$ & $88(96,7 \%)$ & $38(88,4 \%)$ & 0,11 \\
\hline Quantification du tabagisme (en PA) & $37,81 \pm 21,70$ & $40,4 \pm 20,25$ & $28,8 \pm 18,26$ & 0,421 \\
\hline $\begin{array}{l}\text { Exposition professionnelle à des } \\
\text { facteurs inducteurs de BPCO }\end{array}$ & $12(9,0 \%)$ & $10(11,0 \%)$ & $2(4,7 \%)$ & 0,413 \\
\hline Facteur déclenchant les symptômes & & & & $\mathbf{0 , 0 3 1}$ \\
\hline $\begin{array}{ll}\text { - } & \text { Allergènes } \\
\text { - } & \text { Effort physique } \\
\text { - } & \text { Changement de temps }\end{array}$ & $\begin{array}{l}2(1,5 \%) \\
41(30,6 \%) \\
2(1,5 \%)\end{array}$ & $\begin{array}{l}0(0,0 \%) \\
27(29,7 \%) \\
0(0,0 \%)\end{array}$ & $\begin{array}{l}2(11,1 \%) \\
14(77,8 \%) \\
2(11,1 \%)\end{array}$ & \\
\hline $\begin{array}{l}\text { Facteur déclenchant les exacerbations } \\
\text { - } \text { Infectieux } \\
\text { - } \text { Cardiaque } \\
\text { - } \text { Allergène } \\
\text { - } \text { Rupture thérapeutique } \\
\text { - } \quad \text { Chaleur }\end{array}$ & $\begin{array}{l}50(37,3 \%) \\
1(0,7 \%) \\
5(3,7 \%) \\
0 \\
2(1,5 \%)\end{array}$ & $\begin{array}{l}34(85,0 \%) \\
1(2,5 \%) \\
3(7,5 \%) \\
0 \\
1(2,5 \%)\end{array}$ & $\begin{array}{l}16(84,2 \%) \\
0(0,0 \%) \\
2(10,5 \%) \\
0 \\
1(5,3 \%)\end{array}$ & 0,456 \\
\hline Eosinophilie sanguine $\left(/ \mathrm{mm}^{3}\right)$ & $285,89 \pm 129,7$ & $249,0 \pm 167,15$ & $386,7 \pm 226,36$ & 0,017 \\
\hline IgE totales $(\mathrm{kUI} / \mathrm{L})$ & $498,25 \pm 684,74$ & $581,5 \pm 591,84$ & $487,4 \pm 852,16$ & 0,895 \\
\hline
\end{tabular}

Tableau 3 : Facteurs étiologiques de la population en fonction de l'existence d'un ACOS selon le score diagnostique (PA : paquets-années).

En utilisant la définition issue du consensus d'experts, on retrouvait significativement dans le groupe ACOS d'avantage d'atopie et d'allergie, ainsi que des $\operatorname{IgE}$ totales plus élevées. L'absence d'autres facteurs mis en évidence peut s'expliquer par les petits effectifs, diminuant la puissance statistique (Tableau 4). 


\begin{tabular}{|c|c|c|c|c|}
\hline & $\begin{array}{l}\text { Population } \\
\text { générale } \\
(\mathrm{n}=134)\end{array}$ & $\begin{array}{l}\text { Non-ACOS } \\
(\mathrm{n}=118)\end{array}$ & $\begin{array}{l}\text { ACOS } \\
(\mathrm{n}=16)\end{array}$ & $\mathrm{p}$ \\
\hline Age de début des symptômes (années) & $49,64 \pm 19,9$ & $48,74 \pm 20,5$ & $56,5 \pm 13,14$ & 0,171 \\
\hline $\begin{array}{l}\text { Variabilité des symptômes dans la } \\
\text { journée }\end{array}$ & $14(10,4 \%)$ & $12(10,2 \%)$ & $2(12,5 \%)$ & 0,674 \\
\hline Antécédent d'atopie & $18(13,4 \%)$ & $15(12,7 \%)$ & $3(18,8 \%)$ & $\mathbf{0 , 0 1 8}$ \\
\hline Antécédents d'allergie & $28(20,9 \%)$ & $24(20,3 \%)$ & $4(25,0 \%)$ & $\mathbf{0 , 0 2 1}$ \\
\hline Antécédent d'asthme & $37(27,6 \%)$ & $32(27,1 \%)$ & $5(31,3 \%)$ & 0,462 \\
\hline Antécédents familiaux d'asthme & $8(6,0 \%)$ & $7(5,9 \%)$ & $1(6,3 \%)$ & 0,545 \\
\hline Antécédents familiaux d'atopie & $2(1,5 \%)$ & $2(1,7 \%)$ & $0(0 \%)$ & 0,548 \\
\hline $\begin{array}{l}\text { Exposition professionnelle à des } \\
\text { facteurs inducteurs d'asthme }\end{array}$ & $21(15,7 \%)$ & $20(16,9 \%)$ & $1(6,3 \%)$ & 0,200 \\
\hline Tabagisme actif & $126(94 \%)$ & $110(93,2 \%)$ & $16(100 \%)$ & 0,595 \\
\hline Quantification du tabagisme (en PA) & $37,81 \pm 21,70$ & $37,19 \pm 20,84$ & $32,81 \pm 15,81$ & 0,421 \\
\hline $\begin{array}{l}\text { Exposition professionnelle à des } \\
\text { facteurs inducteurs de BPCO }\end{array}$ & $12(9,0 \%)$ & $12(10,2 \%)$ & $0(0,0 \%)$ & 0,178 \\
\hline $\begin{array}{cl}\text { Facteur déclenchant des symptômes } \\
\text { - } & \text { Allergènes } \\
\text { - } & \text { Effort physique } \\
\text { - } & \text { Changement de temps }\end{array}$ & $\begin{array}{l}2(1,5 \%) \\
41(30,6 \%) \\
2(1,5 \%)\end{array}$ & $\begin{array}{l}1(2,7 \%) \\
34(91,9 \%) \\
2(5,4 \%)\end{array}$ & $\begin{array}{l}1(12,5 \%) \\
7(87,5 \%) \\
0(0,0 \%)\end{array}$ & 0,393 \\
\hline $\begin{aligned} & \text { Facteur déclenchant des exacerbations } \\
& \text { - } \text { Infectieux } \\
&- \text { Cardiaque } \\
& \text { - } \text { Allergène } \\
&- \text { Rupture thérapeutique } \\
&- \text { Chaleur }\end{aligned}$ & $\begin{array}{l}50(37,3 \%) \\
1(0,7 \%) \\
5(3,7 \%) \\
0 \\
2(1,5 \%)\end{array}$ & $\begin{array}{l}46(85,2 \%) \\
1(1,9 \%) \\
4(7,4 \%) \\
0 \\
2(3,7 \%)\end{array}$ & $\begin{array}{l}4(80,0 \%) \\
0(0,0 \%) \\
1(20,0 \%) \\
0 \\
0(0,0 \%)\end{array}$ & 0,871 \\
\hline Eosinophilie sanguine $\left(/ \mathrm{mm}^{3}\right)$ & $285,89 \pm 129,7$ & $286,47 \pm 197,73$ & $280 \pm 148,32$ & 0,944 \\
\hline IgE totales $(\mathrm{kUI} / \mathrm{L})$ & $23 \pm 738$ & $266,67 \pm 372,14$ & 2000 & $\mathbf{0 , 0 0 8}$ \\
\hline
\end{tabular}

Tableau 4 : Facteurs étiologiques de la population en fonction de l'existence d'un ACOS selon la définition des experts. PA : paquets-années

\subsubsection{Critères de sévérité}

Plusieurs critères reconnus comme facteurs pronostiques ou indices de gravité ont été utilisés pour caractériser la sévérité de la maladie.

Certains critères ont pu être renseignés chez $100 \%$ des patients : VEMS, stade GOLD, stade mMRC.

D'autres critères n'ont pas été recueillis de façon exhaustive : le test de marche a été réalisé uniquement chez 45 patients sur les $134(33,6 \%)$ et les gaz du sang chez 80 patients soit $59,7 \%$. 
L'indice BODE a été calculé pour tous les patients, mais, intégrant le test de marche, il a pu être sous-estimé chez environ un tiers des patients.

Le nombre d'exacerbations, de consultations et d'hospitalisations ont été renseignés chez $100 \%$ des patients sur les données des comptes-rendus de consultation et d'hospitalisation, avec possiblement une sous-déclaration.

Selon le score diagnostique, les patients présentant un ACOS auraient en moyenne de façon significative un stade GOLD, un score de dyspnée, un indice BODE, une $\mathrm{pCO}_{2}$ et un nombre d'hospitalisation plus bas que les non-ACOS. Il n'a pas été mis en évidence de différence significative concernant le VEMS et la $\mathrm{pO}_{2}$ mais probablement par manque de puissance statistique. Un meilleur VEMS est attendu dans le groupe ACOS dans la mesure où le grade GOLD est meilleur chez les ACOS et qu'il semble exister une tendance à un VEMS plus élevé (Tableau 5).

\begin{tabular}{|c|c|c|c|c|}
\hline & $\begin{array}{l}\text { Population } \\
\text { générale } \\
(\mathrm{n}=134)\end{array}$ & $\begin{array}{l}\text { Non-ACOS } \\
(\mathrm{n}=91)\end{array}$ & $\begin{array}{l}\text { ACOS } \\
(\mathrm{n}=43)\end{array}$ & $\mathrm{p}$ \\
\hline VEMS (en \% des théoriques) & $59,7 \% \pm 15,46$ & $58,2 \% \pm 16,14$ & $62,9 \% \pm 13,52$ & 0,095 \\
\hline Stade GOLD & $2,10 \pm 0,69$ & $2,20 \pm 0,69$ & $1,91 \pm 0,65$ & $\mathbf{0 , 0 2 1}$ \\
\hline Dyspnée stade mMRC & $1,13 \pm 1,12$ & $1,40 \pm 1,18$ & $0,56 \pm 0,70$ & $<0,001$ \\
\hline Test de marche de $6 \mathrm{mn}$ & $76 \% \pm 22,6$ & $73,4 \% \pm 26,14$ & $81,7 \% \pm 10,51$ & 0,260 \\
\hline BODE index & $1,48 \pm 1,6$ & $1,79 \pm 1,70$ & $0,80 \pm 1,14$ & $<0,001$ \\
\hline $\mathrm{pO}_{2}$ & $74,5 \pm 10,9$ & $73,26 \pm 9,82$ & $78,53 \pm 13,51$ & 0,067 \\
\hline $\mathrm{pCO}_{2}$ & $41,2 \pm 6,4$ & $42,1 \pm 6,43$ & $38,4 \pm 5,40$ & $\mathbf{0 , 0 2 7}$ \\
\hline $\begin{array}{l}\text { Nombre d'exacerbations sur } \\
\text { la dernière année }\end{array}$ & $0,8 \pm 1,155$ & $0,81 \pm 1,19$ & $0,77 \pm 1,08$ & 0,832 \\
\hline Nombre de consultations & $0,63 \pm 1,147$ & $0,56 \pm 1,07$ & $0,96 \pm 1,30$ & 0,287 \\
\hline Nombre d'hospitalisations & $0,2 \pm 0,545$ & $0,26 \pm 0,63$ & $0,07 \pm 0,26$ & $\mathbf{0 , 0 1 3}$ \\
\hline $\begin{array}{l}\text { Aggravation au cours des } \\
\text { années }\end{array}$ & $43(32,1 \%)$ & $32(35,2 \%)$ & $11(25,6 \%)$ & 0,324 \\
\hline Mortalité & $0(0,0 \%)$ & $0(0,0 \%)$ & $0(0,0 \%)$ & $\mathrm{NC}$ \\
\hline
\end{tabular}

Tableau 5 : Indices de sévérité de la maladie en fonction de l'existence d'un ACOS selon le score diagnostique. $\mathrm{NC}=$ non calculable

Si l'on utilisait la définition du consensus d'experts, on ne trouvait une différence significative que pour deux critères : le VEMS, plus élevé chez les ACOS, et le nombre d'exacerbations, plus bas chez les ACOS. On trouvait un grade GOLD meilleur et un nombre d'hospitalisations plus bas chez les ACOS avec un «p » à la limite de la significativité. 


\begin{tabular}{|c|c|c|c|c|}
\hline & $\begin{array}{l}\text { Population } \\
\text { générale } \\
(\mathrm{n}=134)\end{array}$ & $\begin{array}{l}\text { Non-ACOS } \\
(\mathrm{n}=118)\end{array}$ & $\operatorname{ACOS}(n=16)$ & $\mathrm{P}$ \\
\hline VEMS (en \% des théoriques) & $59,7 \% \pm 15,46$ & $58,6 \% \pm 15,26$ & $67,9 \% \pm 14,91$ & $\overline{0,024}$ \\
\hline Grade GOLD & $2,10 \pm 0,69$ & $2,14 \pm 0,66$ & $1,81 \pm 0,83$ & 0,069 \\
\hline Dyspnée stade mMRC & $1,13 \pm 1,12$ & $1,16 \pm 1,12$ & $0,88 \pm 1,09$ & 0,340 \\
\hline Test de marche de $6 \mathrm{mn}$ & $76 \% \pm 22,6 \%$ & $75,5 \% \pm 22,97$ & $79,8 \% \pm 22,05$ & 0,694 \\
\hline BODE index & $1,48 \pm 1,6$ & $1,49 \pm 1,63$ & $1,38 \pm 1,50$ & 0,787 \\
\hline $\mathrm{pO}_{2}$ & $74,5 \pm 10,9$ & $74,24 \pm 10,91$ & $77,83 \pm 11,89$ & 0,443 \\
\hline $\mathrm{pCO}_{2}$ & $41,2 \pm 6,4$ & $41,52 \pm 6,44$ & $37,67 \pm 4,27$ & 0,155 \\
\hline $\begin{array}{l}\text { Nombre d'exacerbations sur } \\
\text { la dernière année }\end{array}$ & $0,80 \pm 1,155$ & $0,86 \pm 1,2$ & $0,31 \pm 0,6$ & 0,006 \\
\hline Nombre de consultations & $0,63 \pm 1,147$ & $0,67 \pm 1,18$ & $0,25 \pm 0,62$ & 0,226 \\
\hline Nombre d'hospitalisations & $0,2 \pm 0,545$ & $0,22 \pm 0,57$ & $0,06 \pm 0,25$ & 0,06 \\
\hline $\begin{array}{l}\text { Aggravation au cours des } \\
\text { années }\end{array}$ & $43(32,1 \%)$ & $40(33,9 \%)$ & $3(18,8 \%)$ & 0,268 \\
\hline Mortalité & 0 & 0 & 0 & $\mathrm{NC}$ \\
\hline
\end{tabular}

Tableau 6 : Sévérité en fonction de l'existence d'un ACOS selon la définition des experts (NC $=$ non calculable)

En regroupant par groupe de mauvais pronostic: stades GOLD sévères (stades 3 et 4), dyspnée importante (stades mMRC 3 et 4) et indices BODE de mauvais pronostic (supérieurs à 2), ces trois facteurs (stade GOLD sévère, dyspnée importante et BODE élevé) sont surreprésentés dans le groupe non-ACOS de façon significative avec la définition issue du score diagnostique. En revanche, on trouve autant d'exacerbateurs fréquents dans les deux groupes (tableau 7).

\begin{tabular}{llll}
\hline & $\begin{array}{l}\text { Non-ACOS } \\
(\mathrm{n}=91)\end{array}$ & $\begin{array}{l}\text { ACOS } \\
(\mathrm{n}=43)\end{array}$ & $\mathrm{p}$ \\
\hline BODE $>2$ & $22(24,2 \%)$ & $3(7,0 \%)$ & $\mathbf{0 , 0 1 8}$ \\
$\mathrm{mMRC} \geq 2$ & $38(41,8 \%)$ & $5(11,6 \%)$ & $<\mathbf{0 , 0 0 1}$ \\
Gold $>2$ & $28(30,8 \%)$ & $5(11,6 \%)$ & $\mathbf{0 , 0 1 8}$ \\
Exacerbateurs fréquents & $19(20,2 \%)$ & $6(14,0 \%)$ & 0,47
\end{tabular}

Tableau 7 : Prévalence des stades sévères suivant l'existence d'un ACOS selon le score diagnostique 


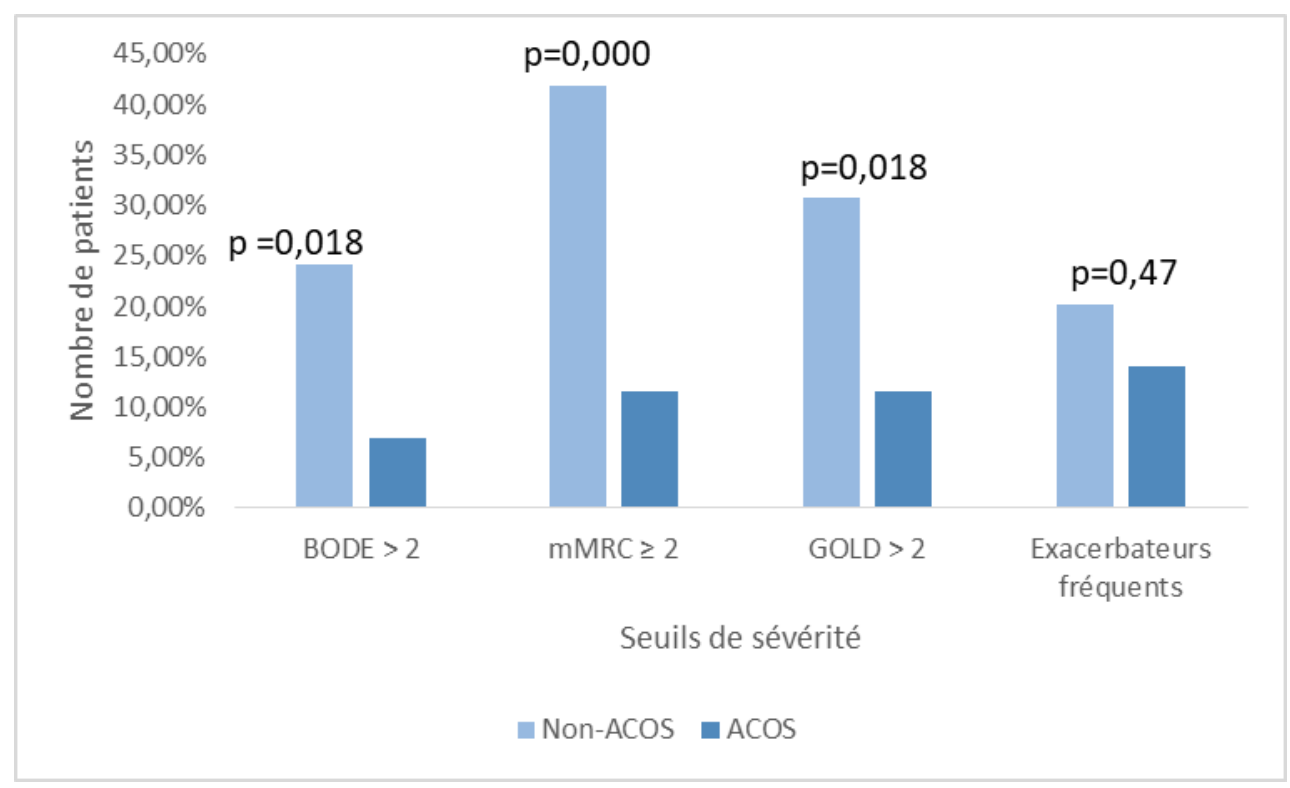

Figure 10 : Fréquence des patients sévères en fonction de l'existence d'un ACOS selon le score diagnostique.

La quasi-totalité des patients sévères étaient en effet compris dans le groupe non-ACOS (figure 11).
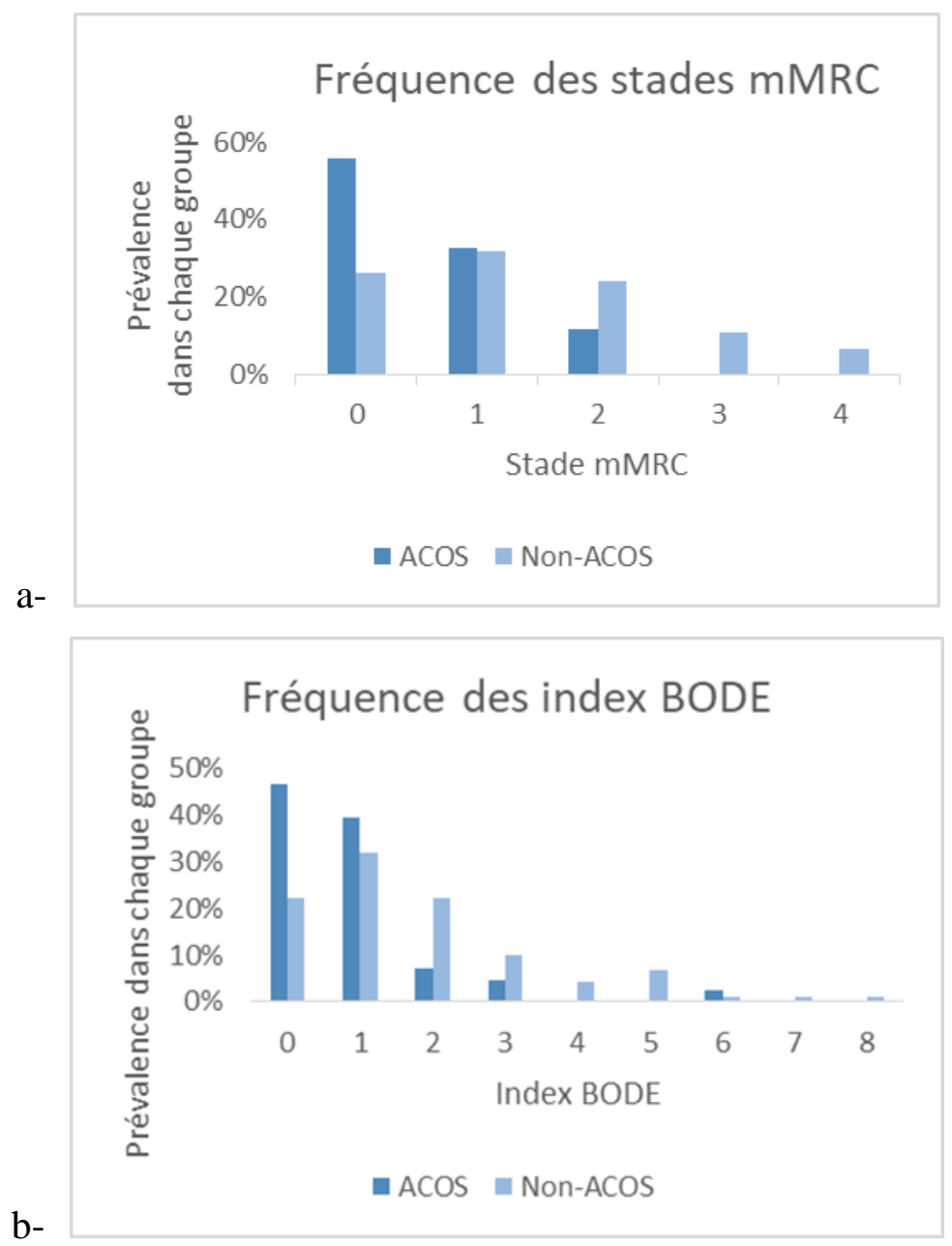

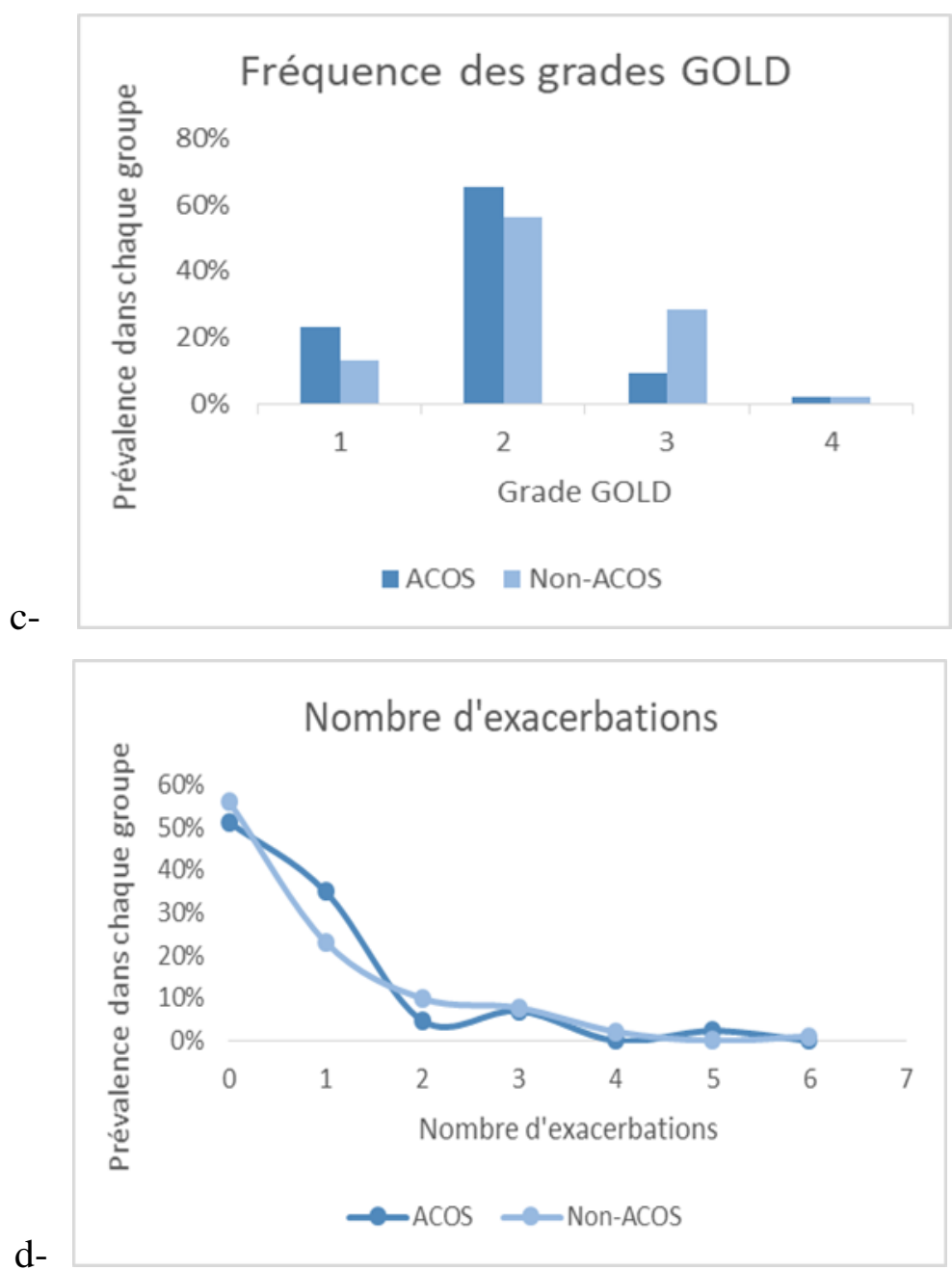

Figure 11 : Fréquence des stades mMRC (a), des index BODE (b), des grades GOLD (c), du nombre d'exacerbations (d) en fonction de la présence d'un ACOS ou non

Si l'on utilisait la définition issue du consensus d'experts, on ne retrouvait pas de différence significative pour ces critères entre les deux groupes, probablement en raison de petits effectifs pour le groupe ACOS (tableau 9).

\begin{tabular}{llll}
\hline & $\begin{array}{l}\text { Non-ACOS } \\
(\mathrm{n}=118)\end{array}$ & ACOS $(\mathrm{n}=16)$ & $\mathrm{P}$ \\
\hline BODE $>2$ & $24(20,3 \%)$ & $1(6,3 \%)$ & 0,304 \\
$\mathrm{mMRC} \geq 2$ & $39(33,1 \%)$ & $4(25,0 \%)$ & 0,58 \\
Gold $>2$ & $31(26,3 \%)$ & $2(12,5 \%)$ & 0,356 \\
Exacerbateurs fréquents & $24(20,3 \%)$ & $1(6,3 \%)$ & 0,304 \\
\hline
\end{tabular}

Tableau 9 : Sévérité en fonction de l'existence d'un ACOS selon la définition des experts 


\subsubsection{Prise en charge thérapeutique}

Les traitements reçus par les patients ont été renseignés dans $100 \%$ des cas à l'aide des comptes rendus de consultation.

En revanche, le soulagement des symptômes par la prise de $\beta$-2-mimétiques d'action rapide était renseigné inconstamment. Avec le score diagnostique, le soulagement des symptômes était renseigné dans $58,4 \%$ des cas d'ACOS et 38,5\% des cas en 1'absence d'ACOS. Avec l'autre définition, il était renseigné pour $37,5 \%$ des patients ACOS et $45,8 \%$ des patients nonACOS.

Si l'on considère les ACOS selon le score diagnostique (tableau 10), on constate une nette prédominance du soulagement des symptômes par les $\beta$-2-mimétiques dans le groupe ACOS, de façon logique dans la mesure où ce critère fait partie du score. Pour les autres traitements, aucune différence n'a été constatée. Les corticoïdes oraux, la théophylline, l'omalizumab, le mépolizumab ou l'azithromycine ne sont pas ou peu prescrits, concernant ainsi de petits effectifs, rendant les analyses statistiques non faisables ainsi que pour la réhabilitation respiratoire, peu prescrite ou rarement renseignée dans les comptes rendus.

En revanche, une différence était attendue pour les corticostéroïdes inhalés mais n'a pas été constatée.

\begin{tabular}{|c|c|c|c|c|}
\hline & $\begin{array}{l}\text { Population } \\
\text { générale } \\
(\mathrm{n}=134)\end{array}$ & $\begin{array}{l}\text { Non-ACOS } \\
(\mathrm{n}=91)\end{array}$ & $\operatorname{ACOS}(n=43)$ & $\bar{p}$ \\
\hline $\begin{array}{l}\text { Soulagement des symptômes par } \\
\text { la prise de } \beta \text {-2-mimétiques }\end{array}$ & $42(31,3 \%)$ & $18(51,4 \%)$ & $24(96,0 \%)$ & $<0,001$ \\
\hline Corticostéroïdes oraux & $3(2,2 \%)$ & $2(2,2 \%)$ & $1(2,2 \%)$ & $\mathrm{NC}$ \\
\hline Corticostéroïdes inhalés & $58(43,3 \%)$ & $39(42,0 \%)$ & $19(44,2 \%)$ & 1,000 \\
\hline$\beta$-2-longue durée d'action & $106(79,3 \%)$ & $72(79,1 \%)$ & $34(79,1 \%)$ & 1,000 \\
\hline Anti-cholinergiques & $100(74,6 \%)$ & $70(76,9 \%)$ & $30(69,8 \%)$ & 0,400 \\
\hline Théophylline & $5(3,7 \%)$ & $3(3,3 \%)$ & $2(4,7 \%)$ & $\mathrm{NC}$ \\
\hline Montelukast & $4(3,0 \%)$ & $1(1,1 \%)$ & $3(7,0 \%)$ & $\mathrm{NC}$ \\
\hline Omalizumab & $1(0,7 \%)$ & $1(1,1 \%)$ & $0(0 \%)$ & $\mathrm{NC}$ \\
\hline Mépolizumab & 0 & 0 & 0 & $\mathrm{NC}$ \\
\hline Azithromycine & $2(1,5 \%)$ & $0(0,0 \%)$ & $2(4,7 \%)$ & $\mathrm{NC}$ \\
\hline Anti-histaminiques & $8(6,0 \%)$ & $3(3,3 \%)$ & $5(11,6 \%)$ & 0,11 \\
\hline $\begin{array}{l}\text { Antécédents de réhabilitation } \\
\text { respiratoire }\end{array}$ & $5(3,7 \%)$ & $4(4,4 \%)$ & $1(2,3 \%)$ & $\mathrm{NC}$ \\
\hline
\end{tabular}

Tableau 10: Thérapeutique en fonction de l'existence d'un ACOS selon le score diagnostique $(N C=$ non calculable $)$

Considérant la définition du consensus d'experts, on observe une différence significative de prescription des $\beta$-2-mimétiques de longue durée d'action, qui sont plus fréquemment prescrits dans le groupe non-ACOS. Il ne semble pas exister de traitement davantage prescrit dans le groupe ACOS. 


\begin{tabular}{lllll}
\hline & $\begin{array}{l}\text { Population générale } \\
(\mathrm{n}=134)\end{array}$ & $\begin{array}{l}\text { Non-ACOS } \\
(\mathrm{n}=118)\end{array}$ & ACOS $(\mathrm{n}=16)$ & $\mathrm{p}$ \\
\hline $\begin{array}{l}\text { Soulagement des symptômes } \\
\text { par la prise de } \beta \text {-2-mimétiques }\end{array}$ & $42(31,3 \%)$ & $37(68,5 \%)$ & $5(83,3 \%)$ & 0,658 \\
Corticostéroïdes oraux & $3(2,2 \%)$ & $3(2,5 \%)$ & $0(0,0 \%)$ & 1,000 \\
Corticostérö̈des inhalés & $58(43,3 \%)$ & $54(45,8 \%)$ & $4(25,0 \%)$ & 0,178 \\
$\beta$-2-longue durée d'action & $106(79,1 \%)$ & $97(82,2 \%)$ & $9(56,3 \%)$ & 0,043 \\
Anti-cholinergiques & $100(74,6 \%)$ & $90(76,3 \%)$ & $10(62,5 \%)$ & 0,235 \\
Théophylline & $5(3,7 \%)$ & $4(3,4 \%)$ & $1(6,3 \%)$ & $\mathrm{NC}$ \\
Montelukast & $4(3 \%)$ & $3(2,5 \%)$ & $1(6,3 \%)$ & $\mathrm{NC}$ \\
Omalizumab & $1(0,7 \%)$ & $1(0,8 \%)$ & $0(0,0 \%)$ & $\mathrm{NC}$ \\
Mépolizumab & $0(0,0 \%)$ & $0(0,0 \%)$ & $0(0,0 \%)$ & $\mathrm{NC}$ \\
Azithromycine & $2(1,5 \%)$ & $2(1,7 \%)$ & $0(0,0 \%)$ & $\mathrm{NC}$ \\
Anti-histaminiques & $8(6 \%)$ & $6(5,1 \%)$ & $2(12,5 \%)$ & $\mathrm{NC}$ \\
Antécédents de réhabilitation & $5(3,7 \%)$ & $5(4,2 \%)$ & $0(0,0 \%)$ & $\mathrm{NC}$ \\
respiratoire & & & & \\
\hline Tableau & & & & \\
\hline
\end{tabular}

Tableau 11 : Thérapeutique en fonction de l'existence d'un ACOS selon la définition des experts

En ce qui concerne la prescription des corticostéroides inhalés (CSI), seulement 29,3\% des patients en recevant étaient exacerbateurs fréquents et parmi les 70,7\% de patients non exacerbateurs sous CSI, seuls $34 \%$ avaient un ACOS selon le score diagnostique. Au total, $44,8 \%$ des patients recevant un CSI étaient ni exacerbateurs fréquents, ni porteurs d'un ACOS.

\subsubsection{Variabilité des EFR sous $\beta$-2-mimétiques}

Parmi les 134 patients, 113 (84,3\%) ont bénéficié d'un test de réversibilité.

Sur les 113 patients, 80 présentaient des EFR strictement non réversibles. Parmi ces patients, 20 présentaient un ACOS selon le score diagnostique, et 9 selon la définition du consensus.

Il existait 33 patients qui présentaient une réversibilité, au moins partielle. Les 7 patients qui présentaient une réversibilité supérieure à $400 \mathrm{~mL}$ étaient également comptés parmi le groupe « réversibilité supérieure à $200 \mathrm{~mL}$ et $12 \%$ ».

Parmi ces 33 patients, il n'a pas été constaté de différence significative entre les groupes ACOS et non-ACOS. Il a seulement été mis en évidence une réversibilité non significative (gain de VEMS > $200 \mathrm{~mL}$ mais < 12\%) significativement plus importante dans le groupe ACOS suivant la définition du score diagnostique (Tableaux 12 et 13).

Ces constatations suggèrent que la réversibilité n'est ni suffisante ni nécessaire au diagnostic d'ACOS. Certains patients non-ACOS semblent présenter une réversibilité, de même que tous les ACOS ne sont pas réversibles. 


\begin{tabular}{lllll}
\hline & $\begin{array}{l}\text { Population générale } \\
(\mathrm{n}=134)\end{array}$ & $\begin{array}{l}\text { Non-ACOS } \\
(\mathrm{n}=91)\end{array}$ & ACOS $(\mathrm{n}=43)$ & $\mathrm{p}$ \\
\hline Réversibilité non significative & $5(3,7 \%)$ & $1(1,1 \%)$ & $4(9,3 \%)$ & $\mathbf{0 , 0 2 6}$ \\
Réversibilité $>200 \mathrm{~mL}$ et $12 \%$ & $28(20,9 \%)$ & $18(19,8 \%)$ & $10(23,3 \%)$ & 0,393 \\
Réversibilité $>400 \mathrm{~mL}$ & $7(5,9 \%)$ & $5(5,5 \%)$ & $2(4,7 \%)$ & 0,514 \\
\hline
\end{tabular}

Tableau 12 : variabilité des EFR sous $\beta$-2-mimétiques en fonction de l'existence d'un ACOS selon le score diagnostique

\begin{tabular}{lllll}
\hline & $\begin{array}{l}\text { Population générale } \\
(\mathrm{n}=134)\end{array}$ & $\begin{array}{l}\text { Non-ACOS } \\
(\mathrm{n}=118)\end{array}$ & ACOS $(\mathrm{n}=16)$ & $\mathrm{p}$ \\
\hline Réversibilité non significative & $5(3,7 \%)$ & $4(3,4 \%)$ & $1(6,3 \%)$ & 0,783 \\
Réversibilité $>200 \mathrm{~mL}$ et $12 \%$ & $28(20,9 \%)$ & $25(21,2 \%)$ & $3(18,8 \%)$ & 0,927 \\
Réversibilité $>400 \mathrm{~mL}$ & $7(5,9 \%)$ & $7(5,9 \%)$ & $0(0 \%)$ & $\mathrm{NC}$ \\
\hline
\end{tabular}

Tableau 13 : variabilité des EFR sous $\beta$-2-mimétiques en fonction de l'existence d'un ACOS selon la définition des experts (NC: non calculable)

\subsubsection{Variabilité des EFR dans l'année}

Parmi les 134 patients, 119 (88,8\%) ont bénéficié de deux EFR dans l'année.

Les patients qui ne présentaient pas de réversibilité étaient au nombre de 60 , soit $44,8 \%$ de la population et se répartissaient de façon égale entre ACOS et non-ACOS quelle que soit la définition utilisée.

Concernant les patients réversibles, il n'a pas été mis en évidence de différence entre les groupes «ACOS » et «non-ACOS » excepté pour le critère " réversibilité supérieure à $400 \mathrm{~mL} »$, cinq fois plus fréquent dans le groupe ACOS (Tableau 14).

Il existe donc une variabilité pour plus d'un patient sur deux au cours de l'année, qu'il soit BPCO non-ACOS ou ACOS. Cette variabilité pourrait être expliquée par un véritable facteur spastique, par des conditions d'examen différentes (par exemple, patient en cours d'exacerbation) ou par une variabilité dans l'exécution de l'EFR.

\begin{tabular}{lllll}
\hline & $\begin{array}{l}\text { Population générale } \\
(\mathrm{n}=134)\end{array}$ & $\begin{array}{l}\text { Non-ACOS } \\
(\mathrm{n}=91)\end{array}$ & $\begin{array}{l}\text { ACOS } \\
(\mathrm{n}=43)\end{array}$ & $\mathrm{p}$ \\
\hline Absence de variabilité des EFR & $60(44,8 \%)$ & $41(45,1 \%)$ & $19(44,2 \%)$ & 0,86 \\
Réversibilité non significative & $9(6,7 \%)$ & $6(6,6 \%)$ & $3(7,0 \%)$ & 0,892 \\
Réversibilité $>200 \mathrm{~mL}$ et $12 \%$ & $50(37,3 \%)$ & $33(36,3 \%)$ & $17(39,5 \%)$ & 0,866 \\
Réversibilité $>400 \mathrm{~mL}$ & $18(13,4)$ & $5(5,5 \%)$ & $13(30,2 \%)$ & $<\mathbf{0 , 0 0 1}$ \\
\hline
\end{tabular}

Tableau 14: variabilité des EFR dans l'année en fonction de l'existence d'un ACOS selon le score diagnostique

Selon la définition issue du consensus, on ne constate pas non plus de réversibilité des EFR alors même qu'il s'agit des critères de la définition, probablement en rapport avec de petits effectifs. 


\begin{tabular}{lllll}
\hline & $\begin{array}{l}\text { Population générale } \\
(\mathrm{n}=134)\end{array}$ & $\begin{array}{l}\text { Non-ACOS } \\
(\mathrm{n}=118)\end{array}$ & $\begin{array}{l}\text { ACOS } \\
(\mathrm{n}=16)\end{array}$ & $\mathrm{p}$ \\
\hline Absence de variabilité des EFR & $60(44,8 \%)$ & $55(46,6 \%)$ & $5(31,5 \%)$ & 0,500 \\
Réversibilité non significative & $9(6,7 \%)$ & $6(5,1 \%)$ & $3(18,8 \%)$ & 0,114 \\
Réversibilité $>200 \mathrm{~mL}$ et $12 \%$ & $50(37,3 \%)$ & $44(37,3 \%)$ & $6(37,5 \%)$ & 0,982 \\
Réversibilité $>400 \mathrm{~mL}$ & $18(13,4)$ & $16(13,6 \%)$ & $2(12,5 \%)$ & 0,980 \\
\hline
\end{tabular}

Tableau 15 : variabilité des EFR dans l'année en fonction de l'existence d'un ACOS selon la définition des experts

\subsubsection{Résultats de l'imagerie}

Tous les patients avaient bénéficié d'une radiographie de thorax. Il existait de façon cohérente une distension thoracique plus importante dans le groupe non-ACOS car c'était un critère du score. Cette différence n'était pas retrouvée dans l'autre définition.

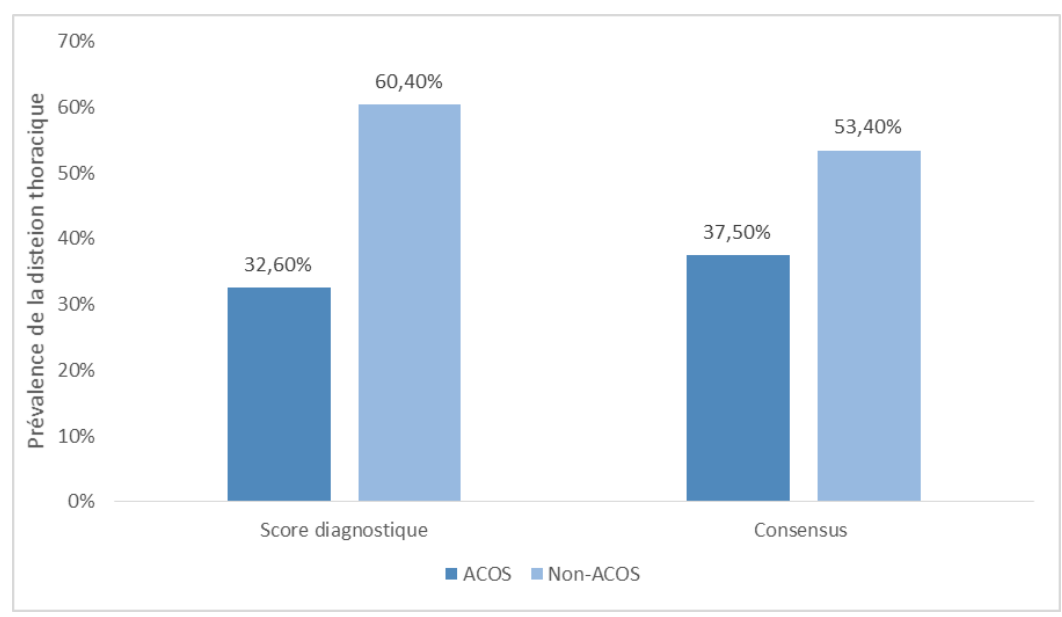

Figure 12 : Distension thoracique en fonction de l'existence d'un ACOS selon les deux définitions.

Concernant le scanner, qui permettait d'éliminer d'éventuels diagnostics associés comme des dilatations des bronches, il a été réalisé chez 109 patients sur 134, soit 81,3\% des patients, sans différence de prescriptions entre les deux groupes.

\subsection{Concordance des définitions}

Parmi les patients ACOS selon le score diagnostique, 6 patients étaient également ACOS avec la seconde définition (14,0\%). Parmi les 16 patients ACOS selon la définition du consensus, seuls $6(37,5 \%)$ l'étaient également avec l'autre définition.

Un test de concordance a été réalisé, montrant une mauvaise corrélation entre les deux définitions, l'indice de corrélation Kappa étant à 0,43. 


\section{DISCUSSION}

Notre étude avait pour but d'identifier les patients susceptibles de répondre à la définition d'ACOS parmi les BPCO du CHU d'Amiens, et d'évaluer les différences entre les 2 groupes, dans l'hypothèse où les ACOS seraient un phénotype de BPCO.

Deux définitions étaient utilisées en l'absence de définition reconnue par l'ensemble de la communauté scientifique.

\subsection{Résultat principal}

La prévalence d'ACOS au sein des patients suivis pour un diagnostic exclusif de BPCO était de $13,28 \%$ selon le score diagnostique, et de 4,61\% selon la définition issue du consensus d'experts. Après exclusion des patients présentant un phénotype d'emphysème et des patients sans trouble ventilatoire obstructif, la prévalence était de 32,1\% des patients selon le score diagnostique et $11,2 \%$ étaient des ACOS possibles selon la définition issue du consensus.

Plusieurs études ont tenté d'évaluer la prévalence des ACOS parmi une population de BPCO ou plus généralement de troubles ventilatoires obstructifs, asthmatiques compris. Aucune n'a utilisé le score diagnostique proposé par l'équipe suisse. Certaines études se basent sur une définition espagnole, pré-existante au consensus d'experts, mais s'en rapprochant. Cette définition pose le diagnostic d'ACOS en cas de deux critères majeurs parmi une réversibilité du VEMS supérieure à $400 \mathrm{~mL}$ et $15 \%$, un antécédent d'asthme ou un NO exhalé $>40 \mathrm{ppb}$, ou d'un critère majeur et deux mineurs parmi des IgE élevées, un antécédent d'atopie ou un gain de VEMS de $12 \%$ et $200 \mathrm{~mL}$.

Parmi les études qui se basent sur cette définition espagnole se trouve l'étude de Cosio (43), qui a évalué la prévalence d'ACOS parmi une cohorte de BPCO dont des patients emphysémateux et a trouvé un chiffre de $15 \%$. Leurs patients BPCO étaient proches de notre population en termes de VEMS, de poids, d'âge et de stade GOLD et BODE mais comprenait plus de femmes et nettement moins de fumeurs (environ un tiers de patients fumeurs actifs contre $94 \%$ dans notre étude), ce qui pourrait expliquer une telle différence de prévalence.

Une autre étude espagnole (47) utilisant ces mêmes critères a trouvé une prévalence de $8,4 \%$, qui se rapproche davantage de notre résultat. Leur population était proche de la nôtre en termes d'âge, de sexe et de tabagisme.

D'autres études, ont utilisé des définitions larges, définissant l'ACOS comme l'existence d'un rapport $\mathrm{VEMS} / \mathrm{CVF}<0,7$ concomitant d'un antécédent de diagnostic d'asthme (51,52) et ont trouvé une prévalence d'ACOS entre 12 et $15 \%$ parmi des cohortes de BPCO. Ces résultats pourraient surestimer la prévalence d'ACOS dans la mesure où les critères sont larges.

Concernant notre population générale, nous avons donc observé un tabagisme et un sexe masculin plus importants que dans les autres études. Nos patients avaient tendance à être plus jeunes dans le groupe ACOS, ce qui apparait être le cas dans l'ensemble des cohortes sur le 
sujet, les ACOS présentant un âge moyen de 59 et 62 ans et les BPCO de 65 à 68 ans (53-55), mais la différence n'était pas significative dans notre étude. Le fait d'observer une prévalence plus basse d'ACOS pourrait être lié à ces différences de population.

Nous avons choisi arbitrairement d'exclure les patients emphysémateux d'une part en l'absence de données existant entre ACOS et emphysème, d'autre part devant la présomption qu'il s'agit d'un phénotype différent. D'abord cliniquement, les emphysémateux sont souvent distingués des autres patients, s'intégrant dans le tableau de pink puffer. Par ailleurs, les études évaluant la répartition des phénotypes de BPCO classent le plus souvent l'emphysème à part $(37,47)$. Elles accentuent l'impression d'un phénotype différent en démontrant une sévérité de la maladie et une surmortalité des emphysémateux par rapport aux autres BPCO (47).

Le fait que notre prévalence d'ACOS soit si basse peut s'expliquer de différentes façons. Premièrement, elle est possiblement réellement plus basse que dans les autres études du même type en raison d'une population différente, et notamment en raison d'un tabagisme plus important dans notre étude. Le tabagisme, par son action inflammatoire via la voie TH1 et l'inflammation neutrophilique, induit un remodelage bronchique et pourrait favoriser certains phénotypes de troubles ventilatoires obstructifs. Autrement, il est possible que la prévalence soit plus basse en raison d'un biais de mesure : certains patients n'ont pas pu être placés dans le groupe ACOS car certaines données étaient manquantes, notamment pour les patients qui n'avaient pas pu bénéficier d'un test de réversibilité et n'ont donc pas pu compléter le 3ème critère majeur (gain $>400 \mathrm{~mL}$ ) ou critère mineur $(>200 \mathrm{~mL}$ et $12 \%$ ), de même pour l'éosinophilie sanguine $>300 / \mathrm{mm} 3$ dans les critères mineurs. Enfin, il est possible que nous ayons choisi une définition trop exigeante ; effectivement, dans la mesure où on reconnait l'asthme comme un trouble ventilatoire obstructif avec gain de $200 \mathrm{~mL}$ et $12 \%$, il semble excessif d'exiger une réversibilité de $400 \mathrm{~mL}$ pour un syndrome de chevauchement asthmeBPCO. La définition espagnole est un peu plus souple, classant la réversibilité supérieure à $400 \mathrm{~mL}$ comme un critère majeur, mais non indispensable au diagnostic d'ACOS.

La prévalence selon la définition du score diagnostique est difficile à comparer car ce score n'a pas été utilisé dans d'autres études pour évaluer la prévalence des ACOS. Une métaanalyse a rassemblé plusieurs études qui évaluaient la prévalence de l'ACOS (56). Les études comprises dans cette méta-analyse avaient des définitions d'ACOS larges, à savoir des caractéristiques d'asthme et de BPCO associées. Leurs résultats d'ACOS sont entre 15 et 55\% de prévalence, et en moyenne de $27 \%$. Là encore, notre prévalence est plus basse, possiblement en raison du tabagisme important, ou en raison d'erreurs dans le recueil de données. En effet, des données étaient manquantes, notamment la variabilité des symptômes dans la journée ou l'année, le facteur déclenchant les symptômes, les antécédents familiaux, l'exposition professionnelle ou domestique, l'efficacité des $\beta$-2-mimétiques, ces critères n'étant soit pas demandés au patient lors de la consultation, soit non renseignés dans le courrier. Par ailleurs, plusieurs critères du score ne sont pas objectifs et laissent une incertitude, dont l'aggravation, qui ne se base pas sur des critères objectifs ainsi que la distension thoracique. Ces données semblent donc importantes dans l'orientation diagnostique et mériteraient d'être systématiquement demandées au patient. 


\subsection{Facteurs étiologiques}

L'antécédent d'asthme était un critère qui faisait partie des deux définitions. Il est donc logique que l'on ait observé presque huit fois plus d'asthmatiques dans le groupe ACOS, ce qui est retrouvé dans la littérature de façon similaire puisque l'antécédent d'asthme est un critère récurrent des définitions.

La variabilité des symptômes et le déclenchement des symptômes par les allergènes étaient également plus importants dans le groupe ACOS, ceci de façon logique puisque ces critères faisaient aussi partie du score.

Les antécédents personnels et familiaux d'asthme ou d'atopie étaient environ trois fois plus fréquents dans le groupe ACOS, dans la mesure où ils faisaient partie du score diagnostique, et ce, de façon cohérente avec la littérature. Une étude suédoise portant sur une cohorte de BPCO a en effet montré deux fois plus d'antécédents familiaux d'asthme chez les ACOS (53).

Concernant l'âge de début des symptômes, il est significativement plus bas dans le groupe ACOS, ce qui est encore le cas dans les autres études du même type, qui trouvent de façon générale des symptômes plus précoces chez les patients ACOS puisque l'asthme avant 40 ans est souvent un des critères diagnostiques $(43,47,51,52)$.

A propos du tabagisme, aucune différence n'a été mise en évidence entre nos deux groupes, contrairement à plusieurs études (53-55) qui trouvaient des patients moins fumeurs.

Concernant les facteurs déclenchants des exacerbations, on ne trouve aucune différence. Les infections apparaissent être le facteur déclenchant le plus fréquent quel que soit le groupe.

Au sujet des marqueurs biologiques, plusieurs études ont été réalisées afin de dessiner des patterns biologiques pouvant expliquer la physiopathologie. Le taux d'éosinophiles sanguins habituellement rapporté dans les groupes ACOS est deux fois plus important que dans les BPCO non-ACOS (43,53), aux alentours de 150 à 200 éosinophiles/mm3 chez les nonACOS, et 270-360/mm3 chez les ACOS, chiffres qui sont proches des nôtres si l'on prend en compte la définition issue du score diagnostique. Il s'agit d'un point intéressant pour plusieurs raisons : n'étant compris dans le score diagnostique, il s'agit d'une réelle différence entre les deux groupes, suggérant l'existence de mécanismes physiopathologiques et inflammatoires réellement différents. Par ailleurs, cela laisse envisager de nouvelles perspectives thérapeutiques chez des patients qui seraient insuffisamment contrôlés ; en premier lieu les corticothérapies inhalée ou orale pourraient trouver leur place, voire l'utilisation des immunothérapies agissant sur la voie des éosinophiles, dont le mépolizumab pour les traitements actuellement commercialisés.

L'éosinophilie dans les expectorations devait également être renseignée mais il n'a pas été possible de recueillir des données, les expectorations étant actuellement envoyés en bactériologie à l'occasion d'exacerbations, où seuls les polynucléaires neutrophiles sont comptés en routine. Cependant, en raison d'un bénéfice des anti-IL5 chez les patients obstructifs ayant plus de 3\% d'éosinophiles dans les expectorations (57), il pourrait être intéressant d'envoyer une expectoration induite en anatomo-pathologie chez les patients qui présentent un tableau de BPCO non typique ou non contrôlée, suspecte d'être intriquée avec 
un asthme, tout d'abord afin de mieux connaître la physiopathologie, et afin d'envisager d'autres thérapeutiques.

De la même façon, le NO exhalé, reflet de l'inflammation bronchique, corrélé à l'éosinophilie sanguine (58), est régulièrement mesuré en excès chez les ACOS $(52,55)$. Il n'a pas été relevé dans notre étude en l'absence de mesure en pratique courante.

Les IgE totales sont, dans ces mêmes cohortes, entre 2 et 3 fois plus élevées dans le groupe ACOS, ce qui a été également observé dans notre étude avec la définition issue du consensus, mais pas dans l'autre définition.

Ces marqueurs biologiques, pour lesquels une différence a été mise en évidence, seraient donc intéressants à rechercher chez tous les BPCO et particulièrement en cas de présentation atypique, d'autant plus qu'il ne s'agit pas de techniques invasives. Il pourrait être proposé de réaliser une NFS et une expectoration induite une fois par an. La NFS permettrait par la même occasion de dépister une anémie participant à la dyspnée ou une polyglobulie secondaire, les expectorations de dépister une colonisation bactérienne ou fongique.

\subsection{Sévérité des patients}

Dans notre étude, quelle que soit la définition utilisée, les patients ACOS semblent moins sévères que les patients non-ACOS.

Selon le score diagnostique, les patients présentant un ACOS auraient en moyenne de façon significative un stade GOLD, un score de dyspnée, un indice BODE, une $\mathrm{pCO}_{2}$ et un nombre d'hospitalisation plus bas que les non-ACOS. Si l'on utilisait la définition du consensus d'experts, on ne trouvait une différence significative que pour deux critères : le VEMS, plus élevé chez les ACOS, et le nombre d'exacerbations, plus bas chez les ACOS. Le stade GOLD et le nombre d'hospitalisations avaient aussi tendance à être meilleurs.

Les BPCO sévères et très sévères (stade GOLD 3 et 4) sont surreprésentés dans le groupe non-ACOS, de même que les indices de BODE > 2, facteur pronostic à partir desquels la mortalité à quatre ans double (59).

Parmi les études qui utilisent la définition espagnole $(43,53)$, proche de celle du consensus, sont également décrits des indices de meilleur pronostic, notamment un grade GOLD léger à modéré, donc un VEMS peu altéré, comme dans notre étude. Elles montrent aussi une meilleure survie, qui n'a pas pu être étudiée dans notre cohorte en l'absence de suivi sur le long terme et de l'absence de décès constaté.

Concernant le VEMS, si l'on observe toujours une valeur plus basse chez les ACOS que les asthmatiques purs $(47,51)$, le rapport avec les BPCO est variable : soit plus bas (47), soit meilleur (51) ou identique pour les patients de la cohorte Initiatives-BPCO (52). Dans notre étude, le VEMS et le grade GOLD sont meilleurs dans le groupe ACOS quelle que soit la définition utilisée, suggérant une réelle différence. L'ensemble des indices pronostiques semble donc converger vers des patients moins graves.

Les patiens ACOS ne sont en revanche pas moins exacerbateurs fréquents que les non-ACOS. S'il s'agissait de rechercher un sous-groupe de patients ACOS nécessitant une prise en charge 
diagnostique et thérapeutique adaptée, il s'agirait donc de cibler ces patients, qui sont les plus graves du groupe. Les exacerbations fréquentes sont en effet associés à une surmortalité, un nombre d'hospitalisations et une qualité de vie moindre $(60,61)$.

Aucun décès n'a été rapporté dans les deux groupes. L'étude n'était pas adaptée à l'évaluation de la survie des patients en raison de l'absence de suivi sur le long terme, mais il paraissait important de pouvoir renseigner d'éventuels décès de patients. Il serait intéressant, avec les groupes définis, de poursuivre le suivi afin de rechercher une différence de mortalité entre les patients ACOS et non-ACOS.

\subsection{Concernant la réversibilité des EFR}

Avec la définition issue du consensus, il n'a pas été mis en évidence de différence de réversibilité sous $\beta$-2-mimétiques entre les deux groupes, possiblement en raison d'effectifs trop faibles dans les groupes ACOS et en dépit du fait qu'il s'agissait d'un critère diagnostique de la définition du consensus.

Selon la définition du score diagnostique, il existe significativement davantage de patients réversibles à plus de $400 \mathrm{~mL}$ dans 1'année dans le groupe ACOS, alors que la variabilité des EFR n'est pas un critère du score. Il existe donc réellement une réversibilité plus fréquente dans le groupe ACOS, avec une variabilité importante. Une forte variabilité des EFR orienterait donc vers un ACOS. Cependant, il existe des patients non-ACOS également réversibles, à moindre degré. Ceci pourrait correspondre soit à des BPCO présentant une réelle réversibilité, soit à une exécution des EFR différentes, notamment pour les patients réalisant leurs premières EFR. La réversibilité, bien qu'elle soit donc plus fréquente en cas d'ACOS, n'est donc pas suffisante au diagnostic d'ACOS.

Dans l'étude de Cosio, qui se basait sur la définition espagnole d'ACOS, on observait trois fois plus de patients réversibles à plus de $200 \mathrm{~mL}$ et $12 \%$ dans le groupe ACOS et un tiers de leurs patients ACOS étaient réversibles à plus de $400 \mathrm{~mL}$, ce qui confirme la tendance des ACOS à être davantage réversibles. Cependant, ne nécessitant qu'un critère majeur et deux mineurs, leurs critères étaient moins exigeants, ce qui pourrait expliquer que nous n'avons pas atteint de telles différences. L'étude française se basant sur la cohorte Initiative-BPCO, qui parlait d'ACOS en cas d'antécédent de diagnostic d'asthme par un médecin, ne montrait pas différence de réversibilité entre les deux groupes, avec un gain de VEMS moyen de $90 \mathrm{~mL}$ dans chaque groupe. Cela montre que la réversibilité n'est pas indispensable au diagnostic d'ACOS.

\subsection{Prise en charge thérapeutique}

Il est difficile d'évaluer les pratiques médicales en l'absence de recommandations actuelles. Une majoration de prescription de corticoïdes inhalés, d'anti-histaminiques, de montelukast et de théophylline pouvait être attendue dans le groupe ACOS dans la mesure où ces traitements sont davantage prescrits chez les patients asthmatiques. Elle est en effet constatée dans 
certaines études, dont la cohorte française $(43,52)$. Cette différence n'a pas été observée, possiblement en raison de petits effectifs gênant l'interprétation des résultats. Néanmoins, en l'absence de syndrome clairement défini actuellement et de recommandations thérapeutiques, il est probable qu'aucune différence n'existe entre les groupes.

Nous nous sommes également intéressés à la prescription de corticostéroïdes inhalés et nous avons mis en évidence que $44,8 \%$ des patients recevant un CSI n'étaient ni exacerbateurs fréquents, ni porteurs d'un ACOS et donc n'avaient pas d'indication pour recevoir un tel traitement. Ces données vont bien dans le sens d'une sur-prescription de CSI constatée sur le plan national.

\subsection{Concordance}

Le coefficient de corrélation de 0,43 est moyen, montrant une faible corrélation entre les deux définitions. Actuellement, aucune des deux ne paraît donc être la définition idéale, la définition du consensus semblant trop exigeante alors que l'autre définition semble imprécise.

\subsection{Forces et limites}

Les limites de notre étude portent sur différents points.

Premièrement, le caractère rétrospectif est à l'origine d'un certain manque de données, parmi lesquelles la réversibilité aux EFR sous $\beta$-2-mimétiques ou dans l'année, l'éosinophilie et les éosinophiles dans les expectorations auraient été parmi les plus intéressants à recueillir.

Deuxièmement, le faible effectif dans certains groupes, lié à un petit nombre d'ACOS avec la seconde définition et à un manque de données, n'a pas permis de conclure sur certains critères.

Enfin, le caractère ponctuel de l'étude a permis de renseigner sur l'état de santé actuel de ces patients et de leurs antécédents. Il aurait cependant été intéressant d'avoir un suivi de plusieurs années notamment pour évaluer l'aggravation dans le temps et la mortalité de chaque groupe.

Néanmoins, il s'agit d'une étude intéressante à plusieurs niveaux. Tout d'abord, elle porte sur la BPCO, un problème de santé publique, pour qui cependant il reste de nombreuses incertitudes quant à la physiopathologie. L'émergence de la notion d'ACOS et de phénotypes de BPCO soulève de nombreuses questions. Il n'existait pas jusqu'à présent d'études évaluant les caractéristiques de la cohorte de BPCO du CHU d'Amiens en ce sens ; et bien que nous n'ayons pas pu conclure sur certains résultats, des éléments sont mis en lumière. On confirme notamment l'hétérogénéité des tableaux de BPCO, et l'existence de patients ayant un syndrome de chevauchement, dont la sévérité et le profil clinique et biologique semblent leur être propres. 
Par ailleurs, nos résultats sont cohérents puisque notre population de BPCO et les patients que nous avons identifiés comme pouvant répondre aux critères d'ACOS ont des caractéristiques phénotypiques proches de celles des autres études européennes du même type.

Bien que les implications dans la prise en charge de nos patients soient encore limitées, il apparait que les patients avec un ACOS présentent autant d'exacerbateurs fréquents que les patients BPCO non-ACOS, et c'est donc à ce sous-groupe que pourraient profiter nos résultats. Ils pourraient en effet bénéficier d'une prise en charge diagnostique plus approfondie et d'une prise en charge thérapeutique plus adaptée si des essais thérapeutiques voyaient le jour. Ce serait également ce groupe qui en tirerait le plus grand bénéfice en termes de santé et de dépenses de santé.

L'étude a en effet permis de mettre en lumière quelques points pouvant justifier d'une amélioration dans la prise en charge de nos malades : réalisation d'EFR avec test de réversibilité plus fréquemment, au moins au diagnostic, et à répéter en cas de présentation atypique, réalisation d'une NFS et de crachats induits (avec mesures des éosinophiles), notamment pour les exacerbateurs fréquents. 
Parmi les patients étiquetés BPCO suivis au CHU d'Amiens, 4 à 13\% des patients semblent présenter un syndrome de chevauchement asthme-BPCO (ACOS). Par rapport aux BPCO non-ACOS, ces patients présentent davantage d'antécédents personnels ou familiaux d'asthme et d'atopie, et leurs symptômes surviennent plus précocement. Ils sont associés à des taux d'éosinophiles et d'IgE sanguins plus élevés. Les indices de sévérité sont en faveur d'une sévérité moindre des patients ACOS mais on compte autant d'exacerbateurs fréquents dans chaque groupe. Comme attendu, la réversibilité des EFR apparaît plus fréquente chez les ACOS mais néanmoins non indispensable au diagnostic d'ACOS. Ces données suggèrent l'existence d'un phénotype de BPCO, de physiopathologie et pronostic différent. Notre étude pourrait être complétée par un suivi sur le long terme afin d'évaluer le pronostic de chaque groupe de patients. Il serait également intéressant de compléter certaines données manquantes: EFR avec test de réversibilité, NFS et crachats induits avec recherche d'éosinophiles. Enfin, la recherche de biomarqueurs serait intéressante afin d'éclairer la physiopathologie : facteurs génétiques, étude du microbiote, analyse des profils inflammatoires locaux. 


\section{ABREVIATIONS}

ACOS : Asthma and COPD Overlap Syndrome

BPCO : BronchoPneumopathie Chronique Obstructive

CAT : COPD Assessment Test

CSI ou ICS : Corticostéroïdes Inhalés

CVF : Capacité Vitale Forcée

EFR : Explorations Fonctionnelles Respiratoires

GINA : Global INitiative for Asthma

GOLD : Global initiative for chronic Obstructive Lung Disease

IL-4, IL-5, IL-8, IL-13 : Interleukines 4, 5, 8, 13

LABA : Bronchodilatateurs $\beta$-2-mimétiques de longue durée d'action

LAMA : Bronchodilatateurs muscariniques (anticholinergiques) de longue durée d'action

NFS : Numération Formule Sanguine

NO : Monoxyde d'azote

PA : Paquet-année

TM6 : Test de Marche de 6mn

TNF : Tumor necrosis factor

TVO : Trouble ventilatoire obstructif

VEMS : Volume Expiratoire Maximal par Seconde 


\section{BIBLIOGRAPHIE}

1. GOLD Guidelines, Guide to COPD diagnostic, managment and prevention, 2018 Report

2. Adeloye D, Chua S, Lee C, Basquill C, Papana A, Theodoratou E, et al. Global and regional estimates of COPD prevalence: Systematic review and meta-analysis. J Glob Health 2015 Dec;5(2):020415.

3. Global, regional, and national age-sex specific all-cause and cause-specific mortality for 240 causes of death, 1990-2013: a systematic analysis for the Global Burden of Disease Study 2013. The Lancet. 10 janv 2015;385(9963):117-71.

4. Bronchite chronique : prévalence et impact sur la vie quotidienne. Analyse des données de l'enquête santé Insee 2002-2003, Institut de Veille Sanitaire.

5. Perez T, Mal H, Aguilaniu B, Brillet P-Y, Chaouat A, Louis R, et al. BPCO et inflammation : mise au point d'un groupe d'experts. Les phénotypes en lien avec l'inflammation. Rev Mal Respir. févr 2011;28(2):192-215.

6. Chinet T, Dumoulin J, Honore I, Braun J-M, Couderc L-J, Febvre M, et al. Place des corticostéroïdes inhalés dans la BPCO. Rev Mal Respir. déc 2016;33(10):877-91.

7. Vestbo J, Hurd SS, Agustí AG, Jones PW, Vogelmeier C, Anzueto A, et al. Global strategy for the diagnosis, management, and prevention of chronic obstructive pulmonary disease: GOLD executive summary. Am J Respir Crit Care Med. 15 févr 2013;187(4):347-65.

8. Hogg JC, Chu F, Utokaparch S, Woods R, Elliott WM, Buzatu L, et al. The nature of smallairway obstruction in chronic obstructive pulmonary disease. N Engl J Med. 24 juin 2004;350(26):2645-53.

9. O'Donnell RA, Peebles C, Ward JA, Daraker A, Angco G, Broberg P, et al. Relationship between peripheral airway dysfunction, airway obstruction, and neutrophilic inflammation in COPD. Thorax. oct 2004;59(10):837-42.

10. Stănescu D, Sanna A, Veriter C, Kostianev S, Calcagni PG, Fabbri LM, et al. Airways obstruction, chronic expectoration, and rapid decline of FEV1 in smokers are associated with increased levels of sputum neutrophils. Thorax. mars 1996;51(3):267-71.

11. Saetta M, Di Stefano A, Turato G, Facchini FM, Corbino L, Mapp CE, et al. CD8+ Tlymphocytes in peripheral airways of smokers with chronic obstructive pulmonary disease. Am J Respir Crit Care Med. mars 1998;157(3 Pt 1):822-6.

12. Papi A, Romagnoli M, Baraldo S, Braccioni F, Guzzinati I, Saetta M, et al. Partial reversibility of airflow limitation and increased exhaled NO and sputum eosinophilia in chronic obstructive pulmonary disease. Am J Respir Crit Care Med. nov 2000;162(5):1773-7.

13. Louis RE, Cataldo D, Buckley MG, Sele J, Henket M, Lau LC, et al. Evidence of mast-cell activation in a subset of patients with eosinophilic chronic obstructive pulmonary disease. Eur Respir J. 1 août 2002;20(2):325-31.

14. Brightling CE, Monteiro W, Ward R, Parker D, Morgan MD, Wardlaw AJ, et al. Sputum eosinophilia and short-term response to prednisolone in chronic obstructive pulmonary disease: a randomised controlled trial. Lancet Lond Engl. 28 oct 2000;356(9240):1480-5. 
15. Snoeck-Stroband JB, Lapperre TS, Gosman MME, Boezen HM, Timens W, Hacken NHT ten, et al. Chronic bronchitis sub-phenotype within COPD: inflammation in sputum and biopsies. Eur Respir J. 1 janv 2008;31(1):70-7.

16. O'Shaughnessy TC, Ansari TW, Barnes NC, Jeffery PK. Inflammation in bronchial biopsies of subjects with chronic bronchitis: inverse relationship of CD8+ T lymphocytes with FEV1. Am J Respir Crit Care Med. mars 1997;155(3):852-7.

17. Vignola AM, Paganin F, Capieu L, Scichilone N, Bellia M, Maakel L, et al. Airway remodelling assessed by sputum and high-resolution computed tomography in asthma and COPD. Eur Respir J. 1 déc 2004;24(6):910-7.

18. Cataldo D, Munaut C, Noël A, Frankenne F, Bartsch P, Foidart JM, et al. MMP-2- and MMP-9linked gelatinolytic activity in the sputum from patients with asthma and chronic obstructive pulmonary disease. Int Arch Allergy Immunol. nov 2000;123(3):259-67.

19. American Thoracic Society, European Respiratory Society. American Thoracic Society/European Respiratory Society statement: standards for the diagnosis and management of individuals with alpha-1 antitrypsin deficiency. Am J Respir Crit Care Med. 1 oct 2003;168(7):818-900.

20. Zervou MI, Tzortzaki EG, Makris D, Gaga M, Zervas E, Economidou E, et al. Differences in microsatellite DNA level between asthma and chronic obstructive pulmonary disease. Eur Respir J. 1 sept 2006;28(3):472-8.

21. Salvi SS, Barnes PJ. Chronic obstructive pulmonary disease in non-smokers. The Lancet. 29 août 2009;374(9691):733-43.

22. Lopez AD, Mathers CD, Ezzati M, Jamison DT, Murray CJL. Global and regional burden of disease and risk factors, 2001: systematic analysis of population health data. Lancet Lond Engl. 27 mai 2006;367(9524):1747-57.

23. Balmes J, Becklake M, Blanc P, Henneberger P, Kreiss K, Mapp C, et al. American Thoracic Society Statement: Occupational contribution to the burden of airway disease. Am J Respir Crit Care Med. 1 mars 2003;167(5):787-97.

24. Ulvestad B, Bakke B, Eduard W, Kongerud J, Lund M. Cumulative exposure to dust causes accelerated decline in lung function in tunnel workers. Occup Environ Med. oct 2001;58(10):663-9.

25. Fairbairn AS, Reid DD. Air Pollution and Other Local Factors in Respiratory Disease. Br J Prev Soc Med. avr 1958;12(2):94-103.

26. Ehrlich RI, White N, Norman R, Laubscher R, Steyn K, Lombard C, et al. Predictors of chronic bronchitis in South African adults. Int J Tuberc Lung Dis Off J Int Union Tuberc Lung Dis. mars 2004;8(3):369-76.

27. Agusti A, Calverley PM, Celli B, Coxson HO, Edwards LD, Lomas DA, et al. Characterisation of COPD heterogeneity in the ECLIPSE cohort. Respir Res. 10 sept 2010;11(1):122.

28. Calverley PMA, Tetzlaff K, Vogelmeier C, Fabbri LM, Magnussen H, Wouters EFM, et al. Eosinophilia, Frequent Exacerbations, and Steroid Response in Chronic Obstructive Pulmonary Disease. Am J Respir Crit Care Med. 17 mars 2017;196(9):1219-21. 
29. Siddiqui SH, Guasconi A, Vestbo J, Jones P, Agusti A, Paggiaro P, et al. Blood Eosinophils: A Biomarker of Response to Extrafine Beclomethasone/Formoterol in Chronic Obstructive Pulmonary Disease. Am J Respir Crit Care Med. 15 août 2015;192(4):523-5.

30. Brusselle GG, Maes T, Bracke KR. Eosinophils in the spotlight: Eosinophilic airway inflammation in nonallergic asthma. Nat Med. août 2013;19(8):977-9.

31. Yayan J, Rasche K. Asthma and COPD: Similarities and Differences in the Pathophysiology, Diagnosis and Therapy. Adv Exp Med Biol. 2016;910:31-8.

32. GINA Guidelines, Global strategy for Asthma management and Prevention (2017 update).

33. Bujarski S, Parulekar AD, Sharafkhaneh A, Hanania NA. The asthma COPD overlap syndrome (ACOS). Curr Allergy Asthma Rep. mars 2015;15(3):509.

34. Sin DD, Miravitlles M, Mannino DM, Soriano JB, Price D, Celli BR, et al. What is asthmaCOPD overlap syndrome? Towards a consensus definition from a round table discussion. Eur Respir J. 2016;48(3):664-73.

35. Marsh SE, Travers J, Weatherall M, Williams MV, Aldington S, Shirtcliffe PM, et al. Proportional classifications of COPD phenotypes. Thorax. sept 2008;63(9):761-7.

36. Soriano JB, Davis KJ, Coleman B, Visick G, Mannino D, Pride NB. The proportional Venn diagram of obstructive lung disease: two approximations from the United States and the United Kingdom. Chest. août 2003;124(2):474-81.

37. Calle Rubio M, Casamor R, Miravitlles M. Identification and distribution of COPD phenotypes in clinical practice according to Spanish COPD Guidelines: the FENEPOC study. Int J Chron Obstruct Pulmon Dis. 9 août 2017;12:2373-83.

38. Menezes AMB, Montes de Oca M, Pérez-Padilla R, Nadeau G, Wehrmeister FC, Lopez-Varela $\mathrm{MV}$, et al. Increased risk of exacerbation and hospitalization in subjects with an overlap phenotype: COPD-asthma. Chest. févr 2014;145(2):297-304.

39. Blanchette CM, Gutierrez B, Ory C, Chang E, Akazawa M. Economic burden in direct costs of concomitant chronic obstructive pulmonary disease and asthma in a Medicare Advantage population. J Manag Care Pharm JMCP. mars 2008;14(2):176-85.

40. Fingleton J, Huang K, Weatherall M, Guo Y, Ivanov S, Bruijnzeel P, et al. Phenotypes of symptomatic airways disease in China and New Zealand. Eur Respir J. déc 2017;50(6).

41. Kitaguchi Y, Komatsu Y, Fujimoto K, Hanaoka M, Kubo K. Sputum eosinophilia can predict responsiveness to inhaled corticosteroid treatment in patients with overlap syndrome of COPD and asthma. Int J Chron Obstruct Pulmon Dis. 2012;7:283-9.

42. Hikichi M, Hashimoto S, Gon Y. Asthma and COPD overlap pathophysiology of ACO. Allergol Int Off J Jpn Soc Allergol. avr 2018;67(2):179-86.

43. Cosio BG, Soriano JB, López-Campos JL, Calle-Rubio M, Soler-Cataluna JJ, de-Torres JP, et al. Defining the Asthma-COPD Overlap Syndrome in a COPD Cohort. Chest. janv 2016;149(1):45-52.

44. Kitaguchi Y, Fujimoto K, Kubo K, Honda T. Characteristics of COPD phenotypes classified according to the findings of HRCT. Respir Med. oct 2006;100(10):1742-52. 
45. Andersén H, Lampela $P$, Nevanlinna A, Säynäjäkangas $O$, Keistinen T. High hospital burden in overlap syndrome of asthma and COPD. Clin Respir J. oct 2013;7(4):342-6.

46. Shaya FT, Dongyi D, Akazawa MO, Blanchette CM, Wang J, Mapel DW, et al. Burden of concomitant asthma and COPD in a Medicaid population. Chest. juill 2008;134(1):14-9.

47. Golpe R, Suárez-Valor M, Martín-Robles I, Sanjuán-López P, Cano-Jiménez E, Castro-Añón O, et al. Mortality in COPD patients according to clinical phenotypes. Int J Chron Obstruct Pulmon Dis. 2018;13:1433-9.

48. Pavord ID, Chanez P, Criner GJ, Kerstjens HAM, Korn S, Lugogo N, et al. Mepolizumab for Eosinophilic Chronic Obstructive Pulmonary Disease. N Engl J Med. 26 2017;377(17):1613-29.

49. Tat TS, Cilli A. Omalizumab treatment in asthma-COPD overlap syndrome. J Asthma Off J Assoc Care Asthma. déc 2016;53(10):1048-50.

50. Valentina Garelli, Nicolas Petitpierre, Laurent P. Nicod. Le syndrome de chevauchement asthme-BPCO. Rev Med Suisse 2015; volume 11.2145-2150

51. Mendy A, Forno E, Niyonsenga T, Carnahan R, Gasana J. Prevalence and Features of AsthmaCOPD Overlap in the U.S. 2007-2012. Clin Respir J. 5 juin 2018

52. Alcázar-Navarrete B, Trigueros JA, Riesco JA, Campuzano A, Pérez J. Geographic variations of the prevalence and distribution of COPD phenotypes in Spain: \&ldquo;the ESPIRAL-ES study\&rdquo;. International Journal of Chronic Obstructive Pulmonary Disease. 2018

53. Ekerljung L, Mincheva R, Hagstad S, Bjerg A, Telg G, Stratelis G, et al. Prevalence, clinical characteristics and morbidity of the Asthma-COPD overlap in a general population sample. $\mathrm{J}$ Asthma Off J Assoc Care Asthma. mai 2018;55(5):461-9.

54. Caillaud D, Chanez P, Escamilla R, Burgel P-R, Court-Fortune I, Nesme-Meyer P, et al. Asthma-COPD overlap syndrome (ACOS) vs «pure » COPD: a distinct phenotype? Allergy. janv 2017;72(1):137-45.

55. Pérez de Llano L, Cosío BG, Miravitlles M, Plaza V, CHACOS study group. Accuracy of a New Algorithm to Identify Asthma-COPD Overlap (ACO) Patients in a Cohort of Patients with Chronic Obstructive Airway Disease. Arch Bronconeumol. avr 2018;54(4):198-204.

56. Alshabanat A, Zafari Z, Albanyan O, Dairi M, FitzGerald JM. Asthma and COPD Overlap Syndrome (ACOS): A Systematic Review and Meta Analysis. PLOS ONE. 3 sept 2015;10(9):e0136065.

57. Nair P, Pizzichini MMM, Kjarsgaard M, Inman MD, Efthimiadis A, Pizzichini E, et al. Mepolizumab for prednisone-dependent asthma with sputum eosinophilia. N Engl J Med. 5 mars 2009;360(10):985-93.

58. Westerhof GA, Korevaar DA, Amelink M, de Nijs SB, de Groot JC, Wang J, et al. Biomarkers to identify sputum eosinophilia in different adult asthma phenotypes. Eur Respir J. sept 2015;46(3):688-96.

59. Celli BR, Cote CG, Marin JM, Casanova C, Montes de Oca M, Mendez RA, et al. The BodyMass Index, Airflow Obstruction, Dyspnea, and Exercise Capacity Index in Chronic Obstructive Pulmonary Disease. N Engl J Med. 4 mars 2004;350(10):1005-12.

60. Gharsalli H, Neffati O, Aouadi S, Bayoudh A, Mokkadem S, Masmoudi M, et al. Caractéristiques des patients BPCO exacerbateurs fréquents. Rev Mal Respir. janv 2015;32:A83. 
61. Oussedik F, Khelafi R, Skander F. Impact des exacerbations de BPCO sur la mortalité. Rev Mal Respir. janv 2016;33:A19. 


\section{Syndromes de chevauchement asthme-BPCO (ACOS) : Etude de la prévalence et des caractéristiques des ACOS parmi la cohorte de BPCO du CHU d'Amiens}

Introduction : Le syndrome de chevauchement asthme-BPCO (ACOS) pourrait être un phénotype de BPCO. L'objectif était d'estimer la prévalence des patients répondant aux critères d'ACOS parmi la cohorte de BPCO du CHU d'Amiens.

Méthodes : Une étude rétrospective a été menée entre mai 2017 et mai 2018 sur la cohorte de BPCO du CHU d'Amiens. Deux définitions étaient utilisées en l'absence de définition consensuelle : un score diagnostique basé sur les critères du GINA, l'autre définition issue d'un consensus d'experts.

Résultats : Sur l'ensemble des patients étiquetés BPCO, 49 (13,3\%) et 17 (4.6\%) patients présentaient un ACOS suivant respectivement la définition du score diagnostique et celle du consensus, prévalence modifiée à $43(32,1 \%)$ et $16(11,2 \%)$ sur 134 patients BPCO après exclusion des patients emphysémateux et des patients n'ayant pas un trouble ventilatoire confirmé. Les patients ACOS étaient moins graves (VEMS à 67,9\% vs 58.6\%, p=0.024, BODE index $0.8 v s 1.79, \mathrm{p}<0.001$ ) mais aussi exacerbateurs fréquents $(\mathrm{p}=0.47)$ que les patients BPCO. Les patients ACOS étaient plus souvent asthmatiques $(\mathrm{p}<0.001)$ et avaient plus souvent des symptômes précoces $(\mathrm{p}<0.001)$, des antécédents personnels $(\mathrm{p}=0.021)$ ou familiaux d'allergie $(\mathrm{p}=0.023)$, une hyperéosinophilie $(\mathrm{p}=0.017)$ ou des $\operatorname{IgE}$ totales élevées $(\mathrm{p}=0.008)$. Aucune différence de réversibilité des EFR n'était constatée sauf pour la réversibilité supérieure à $400 \mathrm{~mL}$, plus fréquente chez les ACOS $(\mathrm{p}<0.001)$.

Conclusion : Parmi les patients suivis pour une BPCO au CHU d'Amiens, 11,2 à 32,1\% répondent aux critères d'ACOS. Il s'agit de patients moins sévères mais tout aussi exacerbateurs fréquents que les $\mathrm{BPCO}$, plus souvent associés à une hyperéosinophilie et à des antécédents personnels ou familiaux d'asthme ou d'allergie. La réversibilité des EFR semble être un critère ni nécessaire ni suffisant pour le diagnostic d'ACOS.

Mots clefs : BPCO ; asthme ; ACOS ; trouble ventilatoire obstructif, phénotypes

\section{Asthma-and-COPD overlap syndrome (ACOS) : Prevalence and characteristics of ACOS among} COPD cohort in Amiens University Hospital.

Introduction: Asthma-and-COPD overlap syndrome (ACOS) could be a COPD phenotype. We aimed to determine prevalence and characteristics of patients who could be ACOS in the Amiens University Hospital COPD cohort.

Methods: We conducted a retrospective study from may 2017 to may 2018 among the Amiens University Hospital COPD cohort. Two definitions were used because of the lack of an official definition: a diagnostic score based on GINA standards, and one diagnostics proposed by an expert consensus.

Results: Among all COPD patients, $49(13,3 \%)$ et 17 (4.6\%) were ACOS respectively following the score definition and the consensus definition, and $43(32,1 \%)$ and $16(11,2 \%)$ among 134 COPD patients after removing emphysematous patients and non-confirmed obstructive trouble. ACOS people were less serious (FEV1: 67,9\% vs 58.6\%, p=0.024, BODE index 0.8 vs 1.79, $\mathrm{p}<0.001$ ) but as frequents exacerbators as non-ACOS COPD patients $(\mathrm{p}=0.47)$. ACOS patients had more often asthma $(\mathrm{p}<0.001)$, early symptoms $(\mathrm{p}<0.001)$, familial $(\mathrm{p}=0.023)$ or personal $(\mathrm{p}=0.021)$ allergy, hypereosinophilia $(\mathrm{p}=0.017)$ or high total $\operatorname{IgE}(\mathrm{p}=0.008)$. No difference was seen in spirometrics except for gain of FEV1 $>400 \mathrm{~mL}$, more frequent in ACOS people $(\mathrm{p}<0.001)$.

Conclusion: Among Amiens University Hospital COPD cohort, 11,2 to 32,1\% of patients can be qualified of ACOS. They are less serious but as frequent exacerbators as COPD. They more often have asthma, early symptoms, familial or personal allergy, hypereosinophilia or high total IgE. Spirometry variation seems not to be necessary nor enough to insure ACOS diagnostic.

Keywords : COPD ; asthma ; ACOS ; obstructive respiratory trouble, phenotype 